\title{
A note on 'Analysis of gamma-ray burst duration distribution using mixtures of skewed distributions'
}

DOI:

10.1093/mnras/stx2373

\section{Document Version}

Final published version

Link to publication record in Manchester Research Explorer

\section{Citation for published version (APA):}

Kwong, H. S., \& Nadarajah, S. (2018). A note on 'Analysis of gamma-ray burst duration distribution using mixtures of skewed distributions'. Monthly Notices of the Royal Astronomical Society, 473(1), 625-646.

https://doi.org/10.1093/mnras/stx2373

\section{Published in:}

Monthly Notices of the Royal Astronomical Society

\section{Citing this paper}

Please note that where the full-text provided on Manchester Research Explorer is the Author Accepted Manuscript or Proof version this may differ from the final Published version. If citing, it is advised that you check and use the publisher's definitive version.

\section{General rights}

Copyright and moral rights for the publications made accessible in the Research Explorer are retained by the authors and/or other copyright owners and it is a condition of accessing publications that users recognise and abide by the legal requirements associated with these rights.

\section{Takedown policy}

If you believe that this document breaches copyright please refer to the University of Manchester's Takedown Procedures [http://man.ac.uk/04Y6Bo] or contact uml.scholarlycommunications@manchester.ac.uk providing relevant details, so we can investigate your claim.

\section{OPEN ACCESS}




\title{
A note on 'Analysis of gamma-ray burst duration distribution using mixtures of skewed distributions'
}

\author{
Hok Shing Kwong and Saralees Nadarajah ${ }^{\star}$ \\ School of Mathematics, University of Manchester, Manchester M13 9PL, UK
}

Accepted 2017 September 11. Received 2017 September 11; in original form 2017 May 29

\begin{abstract}
Tarnopolski [Monthly Notices of the Royal Astronomical Society, 458 (2016) 2024-2031] analysed data sets on gamma-ray burst durations using skew distributions. He showed that the best fits are provided by two skew normal and three Gaussian distributions. Here, we suggest other distributions, including some that are heavy tailed. At least one of these distributions is shown to provide better fits than those considered in Tarnopolski. Five criteria are used to assess best fits.
\end{abstract}

Key words: methods: statistical.

\section{INTRODUCTION}

There have been many studies in grouping of gamma-ray bursts. The gamma-ray bursts can be classified into two groups according to shorter and longer $T_{90}$ durations. But is there a third group? Horvath found evidence of this by fitting a three Gaussian distribution to logarithms of $T_{90}$ for the BATSE 3B data. Mukherjee et al. (1998) used multivariate clustering procedures to discriminate between distinct groups of gamma-ray bursts. The variables burst duration, fluence and spectral hardness were used. They found three groups. But there is no clear rationale for the existence of the third group. It may be influenced strongly by the satellites. Veres et al. (2010) argued that the third group might well be related to the so-called X-ray flashes, but Ripa \& Meszaros (2016) questioned this relation.

Here, we focus on the analysis of data sets relating to gamma-ray burst durations: Savchenko, Neronov \& Courvoisier (2012) found that the durations observed by INTEGRAL have a unimodal distribution; Tarnopolski (2015) modelled the logarithm of $T_{90}$ durations by a mixture of skew distributions; Zitouni et al. (2015) showed that a mixture of three Gaussian distributions fitted the Swift data better than a two Gaussian distribution; for BATSE 4B and current and Swift, Zitouni et al. (2015) found that a three Gaussian distribution followed the observations better than a two Gaussian distribution; Tarnopolski (2016) suggested that mixtures of two non-Gaussian distributions are likely to provide better fits than a three Gaussian distribution if the assumption that data are Gaussian distributions is relaxed. This suggestion was motivated by non-symmetry in the distribution of the logarithm of $T_{90}$ durations due to a non-symmetric distribution of the envelope masses of the progenitors. Tarnopolski (2016), in fact, showed that 'mixtures of two skew normal or two sinh arcsinh distributions are more likely to describe the observed

^E-mail: mbbsssn2@manchester.ac.uk duration distribution of Fermi than a mixture of three standard Gaussian distributions, and that mixtures of two sinh arcsinh or two skew normal distributions are models competing with the conventional three Gaussian distribution in the case of BATSE and Swift'. For others, we refer the readers to Horvath et al. (2008), Zhang \& Choi (2008), Huja, Meszaros \& Ripa (2009), Horvath et al. (2010), Ripa et al. (2012) and Bhave et al. (2017).

The aim of this note is to consider other distributions not considered in the related literature. The distributions considered include both heavy tailed and light tailed. We show that at least one of these distributions provides better fits than the best-fitting distributions noted by Tarnopolski (2016). The data sets from Fermi, BATSE and Swift will be studied.

Section 2 gives a description of the data. Section 3 discusses the distributions, their fitting and the criteria used to select the bestfitting distributions. The results of the fittings and their discussion are provided in Sections 4 and 5. Finally, some conclusions are noted in Section 6.

\section{DATA SETS}

Five data sets ${ }^{1}$ were obtained from three different sources: Fermi, ${ }^{2}$ BATSE $^{3}$ and Swift. ${ }^{4}$ For the Fermi source, three different data sets were considered (i) data from the earliest - 2015 April 29, with outliers excluded; (ii) data from the earliest - 2015 April 29; and (iii) all available data at the time of access. (i) was used in order to make a sensible comparison with results in Tarnopolski (2015).

The following summary statistics of each data set are given in Table 1: minimum, first quartile, median, mean, third quartile,

\footnotetext{
${ }^{1}$ All accessed on 2017 May 10.

2 http://heasarc.gsfc.nasa.gov/W3Browse/fermi/fermigbrst.html

${ }^{3}$ http://gammaray.nsstc.nasa.gov/batse/grb/catalog/

${ }^{4}$ http://swift.gsfc.nasa.gov/archive/grbtext_table/
} 
Table 1. Summary statistics of the data on $\ln T_{90}$.

\begin{tabular}{lccrrr}
\hline Statistics & $\begin{array}{c}\text { Fermi 2015 } \\
\text { (outliers excluded) }\end{array}$ & Fermi 2015 & Fermi 2017 & BATSE & Swift \\
\hline Number of data & 1593 & 1596 & 2074 & 2041 & \multicolumn{1}{c}{914} \\
Minimum & -1.194 & -1.796 & -1.796 & -1.824 & -1.744 \\
Maximum & 2.78 & 2.918 & 2.918 & 3.116 & 3.322 \\
Q1 & 0.735 & 0.730 & 0.751 & 0.350 & 1.021 \\
Median & 1.289 & 1.289 & 1.311 & 1.265 & 1.574 \\
Q3 & 1.685 & 1.685 & 1.675 & 1.692 & 1.949 \\
Mean & 1.122 & 1.120 & 1.125 & 0.993 & 1.407 \\
Skewness & -0.797 & -0.810 & -0.837 & -0.691 & -1.098 \\
Kurtosis & 3.088 & 3.164 & 3.178 & 2.557 & 4.385 \\
Variance & 0.619 & 0.629 & 0.620 & 0.854 & 0.648 \\
Range & 3.975 & 4.714 & 4.714 & 4.940 & 5.067 \\
IQR & 0.949 & 0.954 & 0.925 & 1.341 & 0.928 \\
CV & 0.552 & 0.562 & 0.551 & 0.860 & 0.461 \\
\hline
\end{tabular}

Table 2. Interpretation of AIC.

\begin{tabular}{ll}
\hline$\Delta \mathrm{AIC}$ & Interpretation \\
\hline $0<\Delta \mathrm{AIC} \leq 2$ & Substantial support \\
$4<\Delta \mathrm{AIC} \leq 7$ & Considerably less support \\
$10<\Delta \mathrm{AIC}$ & Essentially no support \\
\hline
\end{tabular}

Table 3. Interpretation of BIC.

\begin{tabular}{ll}
\hline$\Delta$ AIC & Interpretation \\
\hline $0<\Delta$ BIC $\leq 2.2$ & Not worth more than a bare mention \\
$2.2<\Delta$ BIC $\leq 6$ & Positive evidence against \\
$6<\Delta$ BIC $\leq 10$ & Strong evidence against \\
$10<\Delta$ BIC & Very strong evidence against \\
\hline
\end{tabular}

maximum, skewness, kurtosis, variance, coefficient of variation, range and interquartile range.

All data sets are negatively skewed. The skewness is largest for Swift and smallest for BATSE. Four of the data sets have kurtosis greater than that of the Gaussian distribution. The kurtosis is largest for Swift and smallest for BATSE.

The variance is largest for BATSE and smallest for Fermi 2015 with outliers excluded. The range is largest for Swift and smallest for Fermi 2015 with outliers excluded. The interquartile range is largest for BATSE and smallest for Fermi 2017. The coefficient of variation is largest for BATSE and smallest for Swift.

For each data set, we use five different criteria to select the 'best'-fitting distribution. These include the Akaike information criterion (AIC; Akaike 1974), the Bayesian information criterion (BIC; Schwarz 1978), the consistent Akaike information criterion

Table 4. Parameter estimates of the fitted distributions (number of independent parameters in parentheses) to the data set Fermi 2015 with outliers excluded; standard errors are in the scale of $10^{-3}$.

\begin{tabular}{|c|c|c|c|c|c|c|}
\hline $\begin{array}{l}\text { Data: Fermi } 2015 \\
\text { (outliers excluded) }\end{array}$ & $\begin{array}{l}\widehat{\omega_{k}} \\
\text { (s.e.) }\end{array}$ & $\begin{array}{l}\widehat{\mu_{k}} \\
\text { (s.e.) }\end{array}$ & $\begin{array}{l}\widehat{\sigma_{k}} \\
\text { (s.e.) }\end{array}$ & $\begin{array}{c}\widehat{\lambda_{k}} \\
\text { (s.e.) }\end{array}$ & $\begin{array}{l}\widehat{\tau_{k}} \\
(\text { s.e. })\end{array}$ & $\ln L$ \\
\hline Three-Gaussian (8) & $\begin{array}{c}0.108 \\
(0.222) \\
0.266 \\
(0.466) \\
0.626 \\
(0.547)\end{array}$ & $\begin{array}{c}-0.412 \\
(0.926) \\
0.757 \\
(1.387) \\
1.540 \\
(0.397)\end{array}$ & $\begin{array}{c}0.374 \\
(0.503) \\
0.594 \\
(1.293) \\
0.422 \\
(0.250)\end{array}$ & & & -1709.4 \\
\hline Two-Skew normal (7) & $\begin{array}{c}0.205 \\
(0.312) \\
0.795 \\
(0.580)\end{array}$ & $\begin{array}{c}-0.760 \\
(0.876) \\
1.869 \\
(0.371)\end{array}$ & $\begin{array}{c}0.977 \\
(1.590) \\
0.676 \\
(0.411)\end{array}$ & $\begin{array}{c}3.070 \\
(8.794) \\
-1.550 \\
(1.972)\end{array}$ & & -1708.75 \\
\hline Two-Generalized $t$ (9) & $\begin{array}{c}0.404 \\
(0.492) \\
0.596 \\
(0.558)\end{array}$ & $\begin{array}{c}0.557 \\
(0.828) \\
1.504 \\
(0.440)\end{array}$ & $\begin{array}{c}1.485 \\
(0.833) \\
0.656 \\
(0.460)\end{array}$ & $\begin{array}{c}16251.270 \\
(13.442) \\
51.000 \\
(81.077)\end{array}$ & $\begin{array}{c}5.394 \\
(12.881) \\
2.224 \\
(3.080)\end{array}$ & -1707.95 \\
\hline Two-Exponential power (7) & $\begin{array}{c}0.399 \\
(0.495) \\
0.601 \\
(0.482)\end{array}$ & $\begin{array}{c}0.538 \\
(0.832) \\
1.508 \\
(0.434)\end{array}$ & $\begin{array}{c}1.072 \\
(0.609) \\
0.460 \\
(0.317)\end{array}$ & $\begin{array}{c}5.343 \\
(10.830) \\
2.203 \\
(3.509)\end{array}$ & & -1707.89 \\
\hline Two-Skew exponential power (9) & $\begin{array}{c}0.098 \\
(0.212) \\
0.902 \\
(0.603)\end{array}$ & $\begin{array}{c}-0.021 \\
(0.066) \\
1.616 \\
(0.379)\end{array}$ & $\begin{array}{c}0.990 \\
(0.139) \\
0.673 \\
(0.198)\end{array}$ & $\begin{array}{c}0.347 \\
(0.364) \\
0.568 \\
(0.330)\end{array}$ & $\begin{array}{c}4.272 \\
(13.329) \\
1.814 \\
(2.310)\end{array}$ & -1707.17 \\
\hline
\end{tabular}


Table 5. Information criteria of the distributions fitted (number of independent parameters in parentheses) to the data set Fermi 2015 with outliers excluded.

\begin{tabular}{|c|c|c|c|c|c|c|}
\hline $\begin{array}{l}\text { Data: Fermi } 2015 \\
\text { (outliers excluded) }\end{array}$ & $\ln L$ & AIC & $\mathrm{BIC}$ & CAIC & $\mathrm{AICc}$ & HQC \\
\hline Three-Gaussian (8) & -1709.4 & 3434.8 & 3477.78 & 3485.78 & 3434.89 & 3450.76 \\
\hline Two-Generalized $t(9)$ & -1707.95 & 3433.9 & 3482.26 & 3491.26 & 3434.01 & 3451.85 \\
\hline Two-Exponential power (7) & -1707.89 & 3429.78 & 3467.39 & 3474.39 & 3429.85 & 3443.75 \\
\hline Two-Skew exponential power (9) & -1707.17 & 3432.34 & 3480.7 & 3489.7 & 3432.46 & 3450.3 \\
\hline
\end{tabular}

Table 6. Parameter estimates of the fitted distributions (number of independent parameters in parentheses) to the data set Fermi 2015; standard errors are in the scale of $10^{-3}$.

\begin{tabular}{|c|c|c|c|c|c|c|}
\hline Data: Fermi 2015 & $\begin{array}{c}\widehat{\omega_{k}} \\
\text { (s.e.) }\end{array}$ & $\begin{array}{l}\widehat{\mu_{k}} \\
\text { (s.e.) }\end{array}$ & $\begin{array}{c}\widehat{\sigma_{k}} \\
\text { (s.e.) }\end{array}$ & $\begin{array}{c}\widehat{\lambda_{k}} \\
\text { (s.e.) }\end{array}$ & $\begin{array}{c}\widehat{\tau_{k}} \\
\text { (s.e.) }\end{array}$ & $\ln L$ \\
\hline Three-Gaussian (8) & $\begin{array}{c}0.107 \\
(0.227) \\
0.259 \\
(0.488) \\
0.635 \\
(0.550)\end{array}$ & $\begin{array}{c}-0.397 \\
(1.053) \\
0.749 \\
(1.571) \\
1.526 \\
(0.402)\end{array}$ & $\begin{array}{c}0.412 \\
(0.648) \\
0.642 \\
(1.561) \\
0.430 \\
(0.260)\end{array}$ & & & -1728.33 \\
\hline Two-Generalized $t(9)$ & $\begin{array}{c}0.418 \\
(0.518) \\
0.582 \\
(0.559)\end{array}$ & $\begin{array}{c}0.608 \\
(0.870) \\
1.486 \\
(0.461)\end{array}$ & $\begin{array}{c}1.485 \\
(0.877) \\
0.642 \\
(0.491)\end{array}$ & $\begin{array}{c}1.793 \\
(10.145) \\
19110.340 \\
(221.435)\end{array}$ & $\begin{array}{c}7.088 \\
(20.232) \\
2.099 \\
(3.053)\end{array}$ & -1727.74 \\
\hline Two-Skew exponential power (9) & $\begin{array}{c}0.039 \\
(0.170) \\
0.961 \\
(0.620)\end{array}$ & $\begin{array}{c}-0.717 \\
(0.001) \\
1.684 \\
(0.393)\end{array}$ & $\begin{array}{c}0.443 \\
(2.926) \\
0.735 \\
(0.179)\end{array}$ & $\begin{array}{l}0.245 \\
(-) \\
0.630 \\
(337)\end{array}$ & $\begin{array}{c}0.184 \\
(-) \\
1.634 \\
(1.666)\end{array}$ & -1710.16 \\
\hline
\end{tabular}

Table 7. Information criteria of the distributions fitted (number of independent parameters in brackets) to the data set Fermi 2015.

\begin{tabular}{|c|c|c|c|c|c|c|}
\hline Data: Fermi 2015 & $\ln L$ & AIC & $\mathrm{BIC}$ & CAIC & $\mathrm{AICc}$ & HQC \\
\hline Two-Skew normal (7) & -1728.21 & 3470.43 & 3508.05 & 3515.05 & 3470.5 & 3484.4 \\
\hline Two-Exponential power (7) & -1728.48 & 3470.95 & 3508.58 & 3515.58 & 3471.02 & 3484.93 \\
\hline Two-Skew exponential power (9) & -1710.16 & 3438.33 & 3486.71 & 3495.71 & 3438.44 & 3456.3 \\
\hline
\end{tabular}

(CAIC; Bozdogan 1987), the corrected Akaike information criterion (AICc; Hurvich \& Tsai 1989) and the Hannan-Quinn criterion (Hannan \& Quinn 1979). Tarnopolski (2016) used the AIC to discriminate among the fitted distributions.

\section{DISTRIBUTIONS FITTED}

Let $X$ denote a continuous random variable representing the $\ln T_{90}$ duration of gamma-ray burst. Let $f(x)$ denote the probability density function of $X$, taken as a mixture of $K$ distributions. Let $f_{k}(x)$ denote the probability density function of the $k$ th component of $f(x)$. We suppose $X$ can be described by the following five distributions: (i) Mixture of $K$ normal distributions specified by

$f(x)=\sum_{k=1}^{K} \frac{\omega_{k}}{\sqrt{2 \pi \sigma_{k}^{2}}} \exp \left[\frac{-\left(x-\mu_{k}\right)^{2}}{2 \sigma_{k}^{2}}\right]$

with $3 K-1$ independent parameters, where $\mu_{k} \in \boldsymbol{R}$ and $\sigma_{k}>0$.

(ii) Mixture of $K$ skew normal distributions (Azzalini 1985) specified by

$f(x)=\sum_{k=1}^{K} \frac{2 \omega_{k}}{\sigma_{k}} \phi\left(\frac{x-\mu_{k}}{\sigma_{k}}\right) \Phi\left(\lambda_{k} \frac{x-\mu_{k}}{\sigma_{k}}\right)$

with $4 K-1$ independent parameters, where $\phi(\cdot)$ and $\Phi(\cdot)$ denote the probability density function and the cumulative 
Table 8. Parameter estimates of the fitted distributions (number of independent parameters in parentheses) to the data set Fermi 2017; standard errors are in the scale of $10^{-3}$.

\begin{tabular}{|c|c|c|c|c|c|c|}
\hline Data: Fermi 2017 & $\begin{array}{c}\widehat{\omega_{k}} \\
\text { (s.e.) }\end{array}$ & $\begin{array}{c}\widehat{\mu_{k}} \\
\text { (s.e.) }\end{array}$ & $\begin{array}{c}\widehat{\sigma_{k}} \\
\text { (s.e.) }\end{array}$ & $\begin{array}{c}\widehat{\lambda_{k}} \\
\text { (s.e.) }\end{array}$ & $\begin{array}{c}\widehat{\tau_{k}} \\
\text { (s.e.) }\end{array}$ & $\ln L$ \\
\hline Three-Gaussian (8) & $\begin{array}{c}0.103 \\
(0.175) \\
0.259 \\
(0.370) \\
0.638 \\
(0.423)\end{array}$ & $\begin{array}{c}-0.388 \\
(0.813) \\
0.739 \\
(1.252) \\
1.527 \\
(0.298)\end{array}$ & $\begin{array}{c}0.396 \\
(0.506) \\
0.668 \\
(1.223) \\
0.416 \\
(0.188)\end{array}$ & & & -2208.41 \\
\hline Two-Generalized $t(9)$ & $\begin{array}{c}0.413 \\
(0.388) \\
0.587 \\
(0.383)\end{array}$ & $\begin{array}{c}0.586 \\
(0.667) \\
1.505 \\
(0.334)\end{array}$ & $\begin{array}{c}1.432 \\
(0.671) \\
0.608 \\
(0.355)\end{array}$ & $\begin{array}{c}1.471 \\
(5.759) \\
3027.160 \\
(8140.490)\end{array}$ & $\begin{array}{c}7.505 \\
(16.648) \\
2.011 \\
(2.101)\end{array}$ & -2208.08 \\
\hline Two-Skew exponential power (9) & $\begin{array}{c}0.035 \\
(0.128) \\
0.965 \\
(0.478)\end{array}$ & $\begin{array}{c}-0.717 \\
(-) \\
1.677 \\
(0.295)\end{array}$ & $\begin{array}{c}0.440 \\
(2.353) \\
0.736 \\
(0.140)\end{array}$ & $\begin{array}{l}0.250 \\
(-) \\
0.609 \\
(0.256)\end{array}$ & $\begin{array}{c}0.180 \\
(-) \\
1.528 \\
(1.197)\end{array}$ & -2206.39 \\
\hline
\end{tabular}

Table 9. Information criteria of the distributions fitted (number of independent parameters in parentheses) to the data set Fermi 2017.

\begin{tabular}{lccccr}
\hline Data:Fermi 2017 & $\ln L$ & AIC & BIC & CAIC & AICc \\
\hline Three-Gaussian (8) & -2208.41 & 4432.81 & 4477.91 & 4485.91 & 4432.88 \\
Two-Skew normal (7) & -2208.79 & 4431.58 & $\mathbf{4 4 7 1 . 0 4}$ & $\mathbf{4 4 7 8 . 0 4}$ & 4431.63 \\
Two-Generalized $t$ (9) & -2208.08 & 4434.16 & 4484.89 & 4493.89 & 4434.25 \\
Two-Exponential power (7) & -2209.16 & 4432.33 & 4471.79 & 4478.79 & 449.34 \\
Two-Skew exponential power (9) & -2206.39 & $\mathbf{4 4 3 0 . 7 7}$ & 4481.51 & 4490.51 & 4452.38 \\
\hline
\end{tabular}

distribution function of the standard Gaussian distribution, respectively, $\mu_{k}, \lambda_{k} \in \boldsymbol{R}$ and $\sigma_{k}>0$.

(iii) Mixture of $K$ generalized- $t$ distributions (McDonald \& Newey 1988) specified by

$f(x)=\sum_{k=1}^{K} \frac{\omega_{k} \tau_{k}}{2 \sigma_{k} \lambda_{k}^{1 / \tau_{k}} B\left(\lambda_{k}, 1 / \tau_{k}\right)}\left[1+\frac{1}{\lambda_{k}}\left|\frac{x-\mu_{k}}{\sigma_{k}}\right|^{\tau_{k}}\right]^{-\left(\lambda_{k}+1 / \tau_{k}\right)}$

with $5 K-1$ independent parameters, where $\mu_{k} \in \boldsymbol{R}$ and $\tau_{k}, \lambda_{k}$, $\sigma_{k}>0$.

(iv) Mixture of $K$ exponential power distributions (Subbotin 1923) specified by

$f(x)=\sum_{k=1}^{K} \frac{\omega_{k} \lambda_{k}}{2 \sigma_{k} \Gamma\left(1 / \lambda_{k}\right)} \exp \left[-\left(\frac{\left|x-\mu_{k}\right|}{\sigma_{k}}\right)^{\lambda_{k}}\right]$

with $4 K-1$ independent parameters, where $\mu_{k} \in \boldsymbol{R}$ and $\sigma_{k}, \lambda_{k}>0$.

(v) Mixture of $K$ skew exponential power distributions (Zhu \& Zinde-Walsh 2009) specified by

$f(x)=\sum_{k=1}^{K} \omega_{k} f_{k}(x)$ $f_{k}(x)= \begin{cases}\frac{1}{\sigma_{k}} K_{\mathrm{EP}}\left(\tau_{k}\right) \exp \left(-\frac{1}{\tau_{k}}\left|\frac{x-\mu_{k}}{2 \lambda_{k} \sigma_{k}}\right|^{\tau_{k}}\right), & \text { if } x \leq \mu_{k}, \\ \frac{1}{\sigma_{k}} K_{\mathrm{EP}}\left(\tau_{k}\right) \exp \left(-\frac{1}{\tau_{k}}\left|\frac{x-\mu_{k}}{2\left(1-\lambda_{k}\right) \sigma_{k}}\right|^{\tau_{k}}\right), & \text { if } x \leq \mu_{k}\end{cases}$

with $5 K-1$ independent parameters, where $K_{\mathrm{EP}}(\tau)=\frac{1}{2 \tau^{1 / \tau} \Gamma(1+1 / \tau)}$, $\mu_{k} \in \boldsymbol{R}, \tau_{k}, \sigma_{k}>0$ and $0<\lambda_{k}<1$.

Several of these distributions are nested (Cox \& Hinkley 1974): The generalized- $t$ distribution for $\tau=2$ and $\lambda \rightarrow \infty$ is a Gaussian distribution, the skew exponential power distribution for $\lambda=0.5$ is an exponential power distribution, and the exponential power distribution for $\lambda=2$ is a Gaussian distribution, and so on.

Each distribution was fitted by the method of maximum likelihood. That is, if $x_{1}, x_{2}, \ldots, x_{n}$ are independent observations on $X$, then the parameters of each distribution are the values maximizing the likelihood

$L(\boldsymbol{\Theta})=\prod_{i=1}^{n} f\left(x_{i} ; \mathbf{\Theta}\right)$

or the log-likelihood

$\ln L(\boldsymbol{\Theta})=\sum_{i=1}^{n} \ln f\left(x_{i} ; \boldsymbol{\Theta}\right)$, 
Table 10. Parameter estimates of the fitted distributions (number of independent parameters in parentheses) to the data set BATSE; standard errors are in the scale of $10^{-3}$

\begin{tabular}{|c|c|c|c|c|c|c|}
\hline Data: BATSE 2015 & $\begin{array}{c}\widehat{\omega_{k}} \\
\text { (s.e.) }\end{array}$ & $\begin{array}{c}\widehat{\mu_{k}} \\
\text { (s.e.) }\end{array}$ & $\begin{array}{c}\widehat{\sigma_{k}} \\
\text { (s.e.) }\end{array}$ & $\begin{array}{c}\widehat{\lambda_{k}} \\
\text { (s.e.) }\end{array}$ & $\begin{array}{c}\widehat{\tau_{k}} \\
\text { (s.e.) }\end{array}$ & $\ln L$ \\
\hline Three-Gaussian (8) & $\begin{array}{c}0.171 \\
(0.208) \\
0.306 \\
(0.365) \\
0.523 \\
(0.397)\end{array}$ & $\begin{array}{c}-0.454 \\
(0.740) \\
0.752 \\
(1.268) \\
1.606 \\
(0.320)\end{array}$ & $\begin{array}{c}0.474 \\
(0.514) \\
0.736 \\
(1.182) \\
0.382 \\
(0.193)\end{array}$ & & & -2443.47 \\
\hline Two-Generalized $t$ (9) & $\begin{array}{c}0.359 \\
(0.256) \\
0.641 \\
(0.410)\end{array}$ & $\begin{array}{c}-0.032 \\
(0.624) \\
1.569 \\
(0.281)\end{array}$ & $\begin{array}{c}1.057 \\
(0.630) \\
0.564 \\
(0.303)\end{array}$ & $\begin{array}{c}29022.660 \\
(518.995) \\
13705.780 \\
(256.314)\end{array}$ & $\begin{array}{c}2.912 \\
(4.581) \\
1.870 \\
(1.625)\end{array}$ & -2444.03 \\
\hline Two-Skew exponential power (9) & $\begin{array}{c}0.287 \\
(0.248) \\
0.713 \\
(0.426)\end{array}$ & $\begin{array}{c}-0.260 \\
(0.627) \\
1.633 \\
(0.289)\end{array}$ & $\begin{array}{c}0.476 \\
(0.323) \\
0.602 \\
(0.198)\end{array}$ & $\begin{array}{c}0.600 \\
(0.465) \\
0.450 \\
(0.251)\end{array}$ & $\begin{array}{c}2.316 \\
(4.416) \\
1.661 \\
(1.944)\end{array}$ & -2443.22 \\
\hline
\end{tabular}

Table 11. Information criteria of the distributions fitted (number of independent parameters in brackets) to the data set BATSE.

\begin{tabular}{lccccc}
\hline Data: BATSE & $\ln L$ & AIC & BIC & CAIC & AICc \\
\hline Three-Gaussian (8) & -2443.47 & 4902.94 & 4947.91 & 4955.91 & 4903.01 \\
Two-Skew normal (7) & -2444.53 & 4903.07 & 4942.41 & 4949.41 & 4903.12 \\
Two-Generalized $t$ (9) & -2443.63 & 4905.27 & 4955.86 & 4964.86 & 4905.35 \\
Two-Exponential power (7) & -2443.44 & $\mathbf{4 9 0 0 . 8 7}$ & $\mathbf{4 9 4 0 . 2 2}$ & $\mathbf{4 9 4 7 . 2 2}$ & 4917.5 \\
Two-Skew exponential power (9) & -2443.22 & 4904.45 & 4955.04 & 4964.04 & 4923.82 \\
\hline
\end{tabular}

where $\boldsymbol{\Theta}=\left(\theta_{1}, \theta_{2}, \ldots, \theta_{k}\right)^{\prime}$ is a vector of parameters specifying $f(\cdot)$. Let $\widehat{\boldsymbol{\Theta}}=\left(\widehat{\theta_{1}}, \hat{\theta_{2}}, \ldots, \hat{\theta_{k}}\right)^{\prime}$ denote the maximum-likelihood estimate of $\boldsymbol{\Theta}$.

The expectation maximization (EM) algorithm (Dempster, Laird \& Rubin 1977) was used for maximum-likelihood estimation. The following procedure was followed:

(i) E-step: set

$$
\omega_{i, k}=\frac{f_{k}\left(x_{i} ; \boldsymbol{\Theta}\right)}{\sum_{m=1}^{K} f_{m}\left(x_{i} ; \boldsymbol{\Theta}\right)}
$$

and

$\omega_{k}=\frac{\sum_{i=1}^{n} \omega_{i, k}}{n}$.

(ii) M-step: set

$$
\widehat{\Theta}=\underset{\Theta}{\operatorname{argmax}} \sum_{k=1}^{K} \sum_{i=1}^{n} \omega_{i, k} \ln f_{k}\left(x_{i} ; \boldsymbol{\Theta}\right)
$$

and

$\widehat{\boldsymbol{\Theta}_{k}}=\underset{\boldsymbol{\Theta}_{k}}{\operatorname{argmax}} \sum_{i=1}^{n} \omega_{i, k} \ln f_{k}\left(x_{i} ; \boldsymbol{\Theta}\right)$.

(iii) Repeat E-step with $\boldsymbol{\Theta}=\widehat{\boldsymbol{\Theta}}$.

(iv) Keep repeating until $[\ln L(\widehat{\boldsymbol{\Theta}})-\ln L(\boldsymbol{\Theta})] \rightarrow 0$.

The M-step was performed using the routine optim in the $\mathrm{R}$ software package (R Development Core Team 2017). The standard errors of $\widehat{\boldsymbol{\Theta}}$ were computed by approximating the covariance matrix of $\widehat{\boldsymbol{\Theta}}$ by the inverse of the observed information matrix, i.e.

$$
\left.\operatorname{cov}(\widehat{\boldsymbol{\Theta}}) \approx\left(\begin{array}{llll}
\frac{\partial^{2} \ln L}{\partial \theta_{1}^{2}} & \frac{\partial^{2} \ln L}{\partial \theta_{1} \partial \theta_{2}} & \cdots & \frac{\partial^{2} \ln L}{\partial \theta_{1} \partial \theta_{k}} \\
\frac{\partial^{2} \ln L}{\partial \theta_{2} \partial \theta_{1}} & \frac{\partial^{2} \ln L}{\partial \theta_{2}^{2}} & \cdots & \frac{\partial^{2} \ln L}{\partial \theta_{2} \partial \theta_{k}} \\
\vdots & \vdots & \ddots & \vdots \\
\frac{\partial^{2} \ln L}{\partial \theta_{k} \partial \theta_{1}} & \frac{\partial^{2} \ln L}{\partial \theta_{k} \partial \theta_{2}} & \cdots & \frac{\partial^{2} \ln L}{\partial \theta_{k}^{2}}
\end{array}\right)^{-1}\right|_{\Theta=\widehat{\Theta}}
$$

Many of the fitted distributions are not nested. Discrimination among them was performed using various criteria: 
Table 12. Parameter estimates of the fitted distributions (number of independent parameters in parentheses) to the data set Swift; standard errors are in the scale of $10^{-3}$.

\begin{tabular}{|c|c|c|c|c|c|c|}
\hline Data: Swift 2015 & $\begin{array}{c}\widehat{\omega_{k}} \\
\text { (s.e.) }\end{array}$ & $\begin{array}{c}\widehat{\mu_{k}} \\
\text { (s.e.) }\end{array}$ & $\begin{array}{c}\widehat{\sigma_{k}} \\
\text { (s.e.) }\end{array}$ & $\begin{array}{c}\widehat{\lambda_{k}} \\
\text { (s.e.) }\end{array}$ & $\begin{array}{c}\widehat{\tau_{k}} \\
\text { (s.e.) }\end{array}$ & $\ln L$ \\
\hline Three-Gaussian (8) & $\begin{array}{c}0.078 \\
(0.330) \\
0.696 \\
(0.319) \\
0.226 \\
(0.305)\end{array}$ & $\begin{array}{c}-0.531 \\
(2.269) \\
1.444 \\
(0.912) \\
1.960 \\
(1.240)\end{array}$ & $\begin{array}{c}0.479 \\
(1.639) \\
0.597 \\
(0.790) \\
0.290 \\
(0.611)\end{array}$ & & & -989.78 \\
\hline Two-Generalized $t(9)$ & $\begin{array}{c}0.224 \\
(0.728) \\
0.776 \\
(1.037)\end{array}$ & $\begin{array}{c}0.579 \\
(2.498) \\
1.635 \\
(0.739)\end{array}$ & $\begin{array}{c}1.782 \\
(2.521) \\
0.793 \\
(0.781)\end{array}$ & $\begin{array}{c}4.624 \\
(144.547) \\
22.920 \\
(752.398)\end{array}$ & $\begin{array}{c}5.787 \\
(39.076) \\
2.427 \\
(5.126)\end{array}$ & -996.653 \\
\hline Two-Skew exponential power (9) & $\begin{array}{c}0.056 \\
(0.286) \\
0.944 \\
(0.279)\end{array}$ & $\begin{array}{c}-0.369 \\
(1.861) \\
1.848 \\
(0.656)\end{array}$ & $\begin{array}{c}0.788 \\
(1.864) \\
0.664 \\
(0.337)\end{array}$ & $\begin{array}{c}0.357 \\
(1.317) \\
0.582 \\
(0.559)\end{array}$ & $\begin{array}{c}1.979 \\
(16.118) \\
1.750 \\
(3.823)\end{array}$ & -989.527 \\
\hline
\end{tabular}

Table 13. Information criteria of the distributions fitted (number of independent parameters in parentheses) to the data set Swift.

\begin{tabular}{llccccc}
\hline Data: Swift & $\ln L$ & AIC & BIC & CAIC & AICc & HQC \\
\hline Three-Gaussian (8) & -989.78 & $\mathbf{1 9 9 5 . 5 6}$ & 2034.1 & 2042.1 & $\mathbf{1 9 9 5 . 7 2}$ \\
Two-Skew normal (7) & -991.112 & 1996.22 & $\mathbf{2 0 2 9 . 9 5}$ & $\mathbf{2 0 3 6 . 9 5}$ & 2010.27 \\
Two-Generalized $t$ (9) & -996.653 & 2011.31 & 2054.67 & 2063.67 & 2096.35 & $\mathbf{2 0 0 9 . 1}$ \\
Two-Exponential power (7) & -996.675 & 2007.35 & 2041.07 & 2048.07 & 2007.47 & 2027.86 \\
Two-Skew exponential power (9) & -989.527 & 1997.05 & 2040.41 & 2049.41 & 1997.25 \\
\hline
\end{tabular}

(i) The AIC due to Akaike (1974) is defined by

$\mathrm{AIC}=2 k-2 \ln L(\widehat{\boldsymbol{\Theta}})$.

The relative probability of a fitted distribution is defined by

$\operatorname{Pr}=\exp \left(\frac{\mathrm{AIC}_{\min }-\mathrm{AIC}}{2}\right)$.

A general rule to interpret the AIC (Burnham \& Anderson 2004) is shown in Table 2.

(ii) The BIC due to Schwarz (1978) is defined by

$\mathrm{BIC}=k \ln n-2 \ln L(\widehat{\boldsymbol{\Theta}})$.

A general rule to interpret the BIC (Raftery 1996) is shown in Table 3.

(iii) The CAIC due to Bozdogan (1987) is defined by

$\mathrm{CAIC}=-2 \ln L(\widehat{\boldsymbol{\Theta}})+k(\ln n+1)$.

The CAIC is similar to the BIC. It penalizes extra parameters harder than BIC does. The interpretation of $\triangle \mathrm{CAIC}$ should be roughly the same as that described in Table 3. (iv) The AICc (Hurvich \& Tsai 1989) is defined by

$\mathrm{AICc}=\mathrm{AIC}+\frac{2 k(k+1)}{n-k-1}$.

The AICc should be used when $n / k<40$ (Burnham \& Anderson 2004). The interpretation of $\triangle \mathrm{AICc}$ should be the same as that described in Table 2.

(v) The Hannan-Quinn criterion (Hannan \& Quinn 1979) is defined by

$\mathrm{HQC}=-2 \ln L(\widehat{\boldsymbol{\Theta}})+2 k \ln \ln n$.

The smaller the values of these criteria, the better the fit. For a more detailed discussion on these criteria, see Burnham \& Anderson (2004) and Fang (2011).

\section{RESULTS}

The five distributions described in Section 3 were fitted to the data described in Section 2. The method of maximum likelihood was used. For all five data sets examined, there is strong evidence (in terms of the information criteria) that the optimal value of $K$ for 


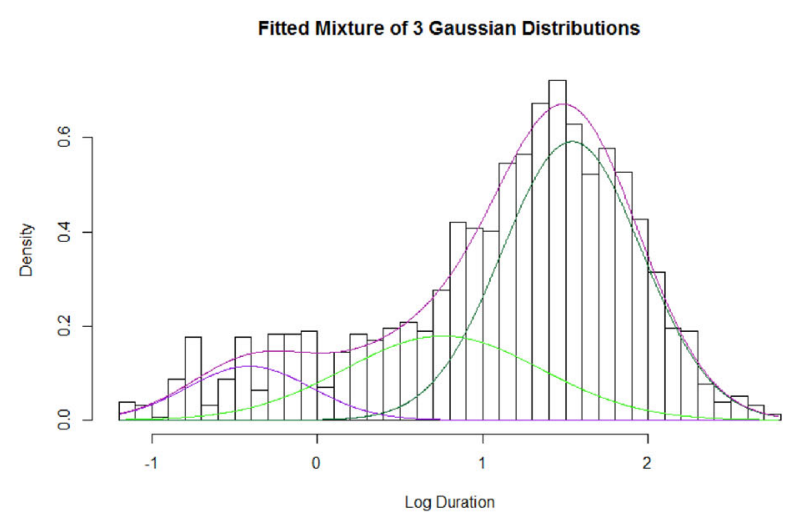

Fitted Mixture of 2 Generalized t Distributions

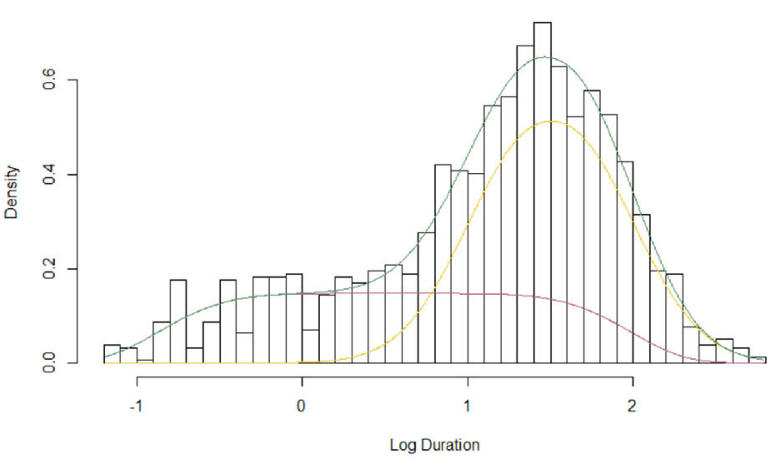

Fitted Mixture of 2 Skewed Exponential Power Distributions

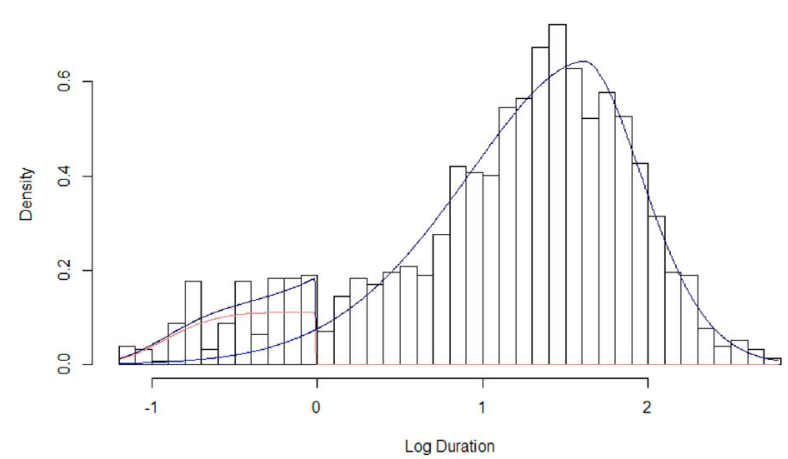

Fitted mixture of 2 Skewed Normal Distribution

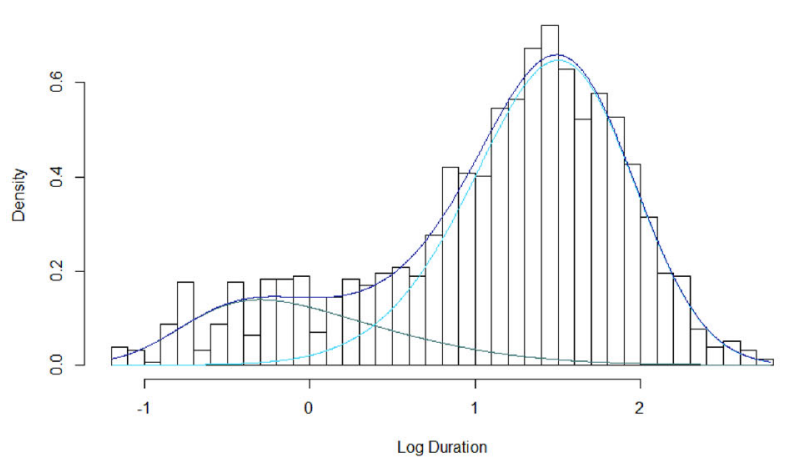

Fitted Mixture of 2 Exponential Power Distributions

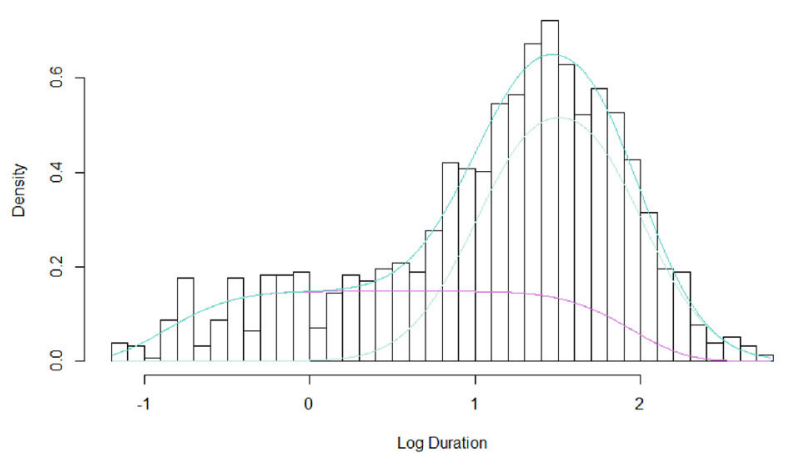

Figure 1. Density of the distributions fitted to the data set Fermi 2015 with outliers excluded.

the skew normal mixture, generalized- $t$ mixture, exponential power mixture and skew exponential power mixture distributions is equal to 2. The optimal value of $K$ for the Gaussian mixture distribution is 3 . The parameter estimates and information criteria for each mixture distribution are presented in Tables 4-13, where the smallest values of the information criteria are given in bold.

For Fermi 2015 with outliers excluded, the parameter estimates and information criteria are shown in Tables 4 and 5, respectively. Density plots, QQ-plots and PP-plots of the fitted distributions are shown in Figs 1, 6 and 11, respectively. For Fermi 2015, the parameter estimates and information criteria are shown in Tables 6 and 7, respectively. The density plot, QQ-plot and PP-plot are shown in Figs 2, 7 and 12, respectively. For Fermi 2017, the parameter estimates and information criteria are shown in Tables 8 and 9, respectively. The density plot, QQ-plot and PP-plot are shown in Figs 3,8 and 13, respectively. For BATSE, the parameter estimates and information criteria are shown in Tables 10 and 11, respectively. The density plot, QQ-plot and PP-plot are shown in Figs 4, 9 and 14, respectively. For Swift, the parameter estimates and information criteria are shown in Tables 12 and 13, respectively. The density plot, QQ-plot and PP-plot are shown in Figs 5, 10 and 15, respectively.

\section{DISCUSSION}

\subsection{Fermi}

\subsubsection{Fermi 2015, outliers excluded}

For this data set, a two exponential power distribution fits best in terms of all five information criteria. There is very strong evidence $(\triangle \mathrm{BIC}, \triangle \mathrm{CAIC}>10)$ and strong evidence $(\Delta \mathrm{AIC}, \Delta \mathrm{AICc}>4)$ 


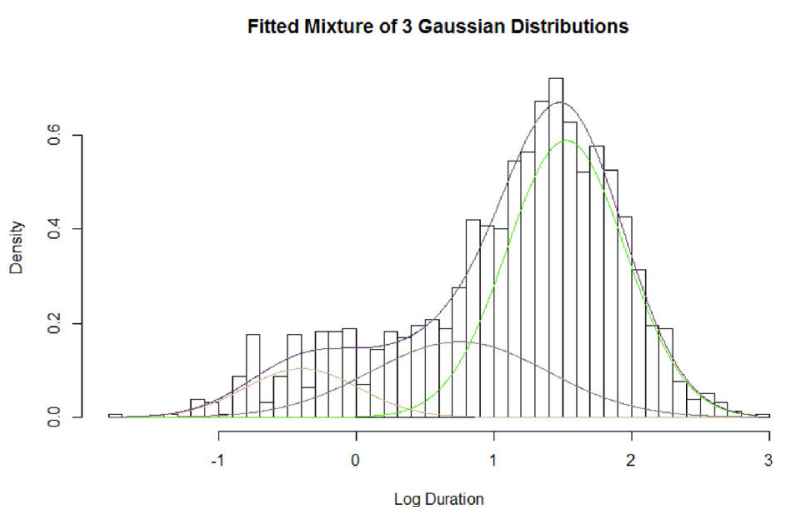

Fitted Mixture of 2 Generalized $t$ Distributions

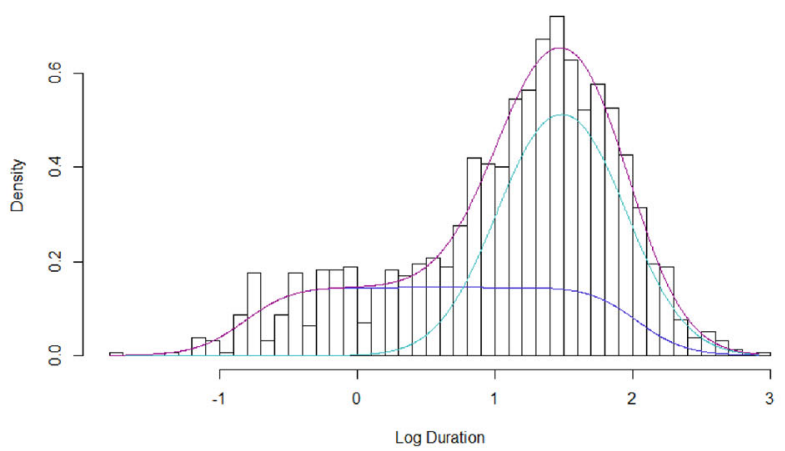

Fitted Mixture of 2 Skewed Exponential Power Distributions

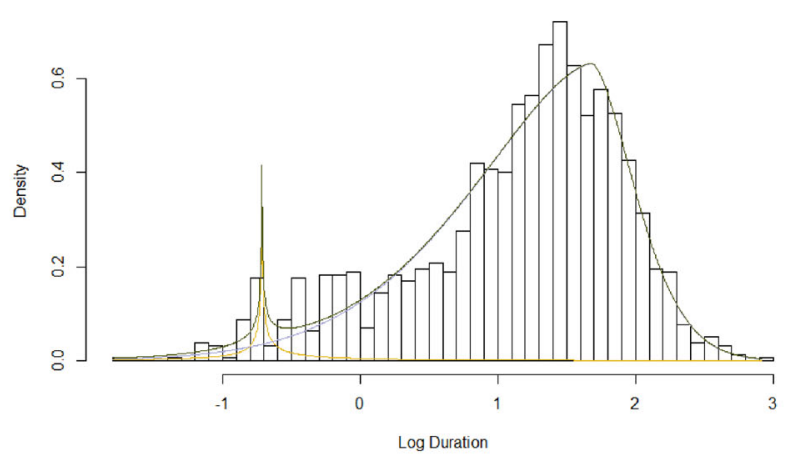

Fitted mixture of 2 Skewed Normal Distribution

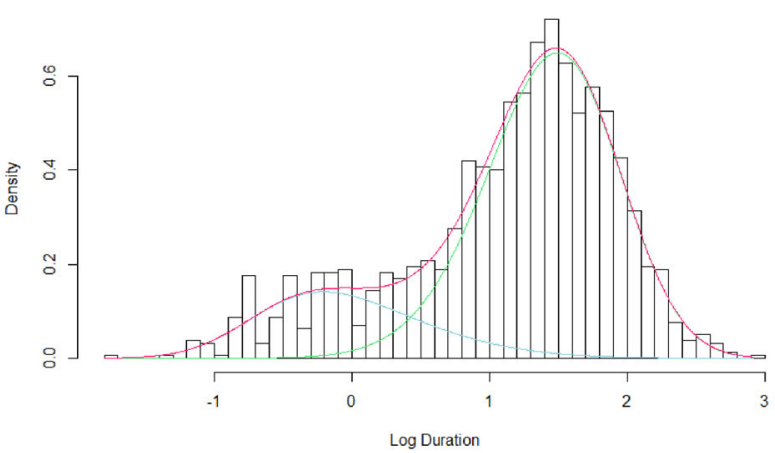

Fitted Mixture of 2 Exponential Power Distributions

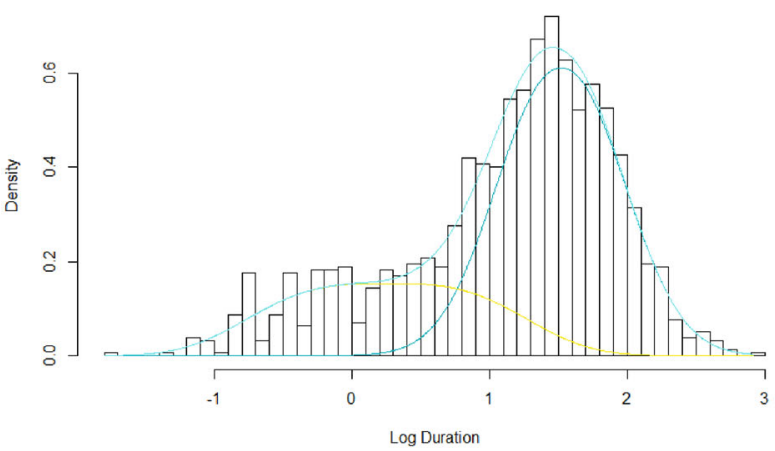

Figure 2. Density of the distributions fitted to the data set Fermi 2015.

that the two exponential power distribution fits better than the three Gaussian distribution. There is also very strong evidence $(\triangle \mathrm{BIC}$, $\Delta \mathrm{CAIC}>10$ ) that the former fits better than the two generalized- $t$ distribution and the two skew exponential power distribution. The values of all of the information criteria for the two skew normal distribution are slightly higher than those for the two exponential power distribution. Although the two exponential power distribution is nested in the two skew exponential power distribution, the extra two parameters used in the two skew exponential power distribution increases the value of $\ln L$ by only 0.72 . This suggests the possibility that the distribution of Fermi $T_{90}$ can be modelled by a mixture of platykurtic distributions rather than a mixture of skew distributions. Each mixture component of the two exponential power distribution has a kurtosis less than that of the Gaussian distribution, meaning each component has heavier tails. The component having the larger weight has the smaller scale and larger kurtosis. It is located to the right-hand side of the component having the smaller weight. In terms of QQ-plot and PP-plot, the three Gaussian, two skew normal, two generalized- $t$ and two exponential power distributions fit the data very well. The two skew exponential power distribution fits the data slightly worse than the other four.

\subsubsection{Fermi 2015}

For this data set, the results obtained were quite different from those in Section 5.1.1. There is very strong evidence ( $\triangle \mathrm{AIC}, \triangle \mathrm{BIC}$, $\triangle \mathrm{CAIC}, \triangle \mathrm{AICc}, \triangle \mathrm{HQC}>10)$ that the two skew exponential power distribution describes the data better than the other four distributions. However, the density plot, QQ-plot and PP-plot show an unusual fit to the left half of the distribution. We argue that 

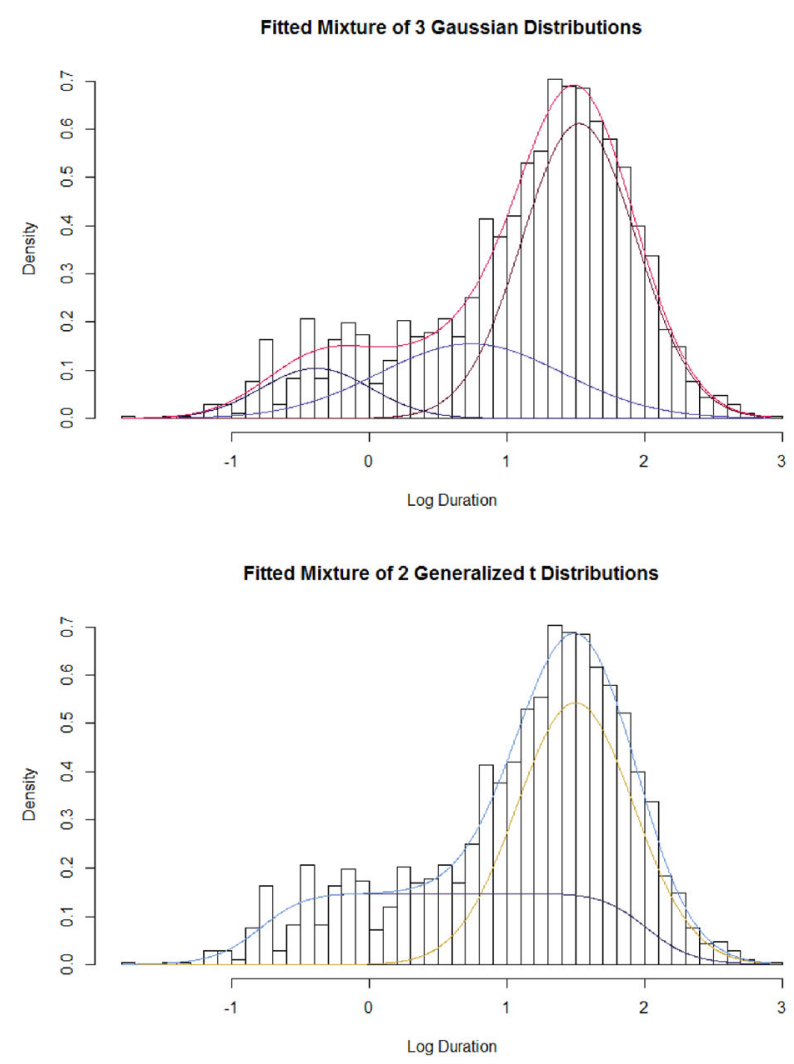

Fitted Mixture of 2 Skewed Exponential Power Distributions

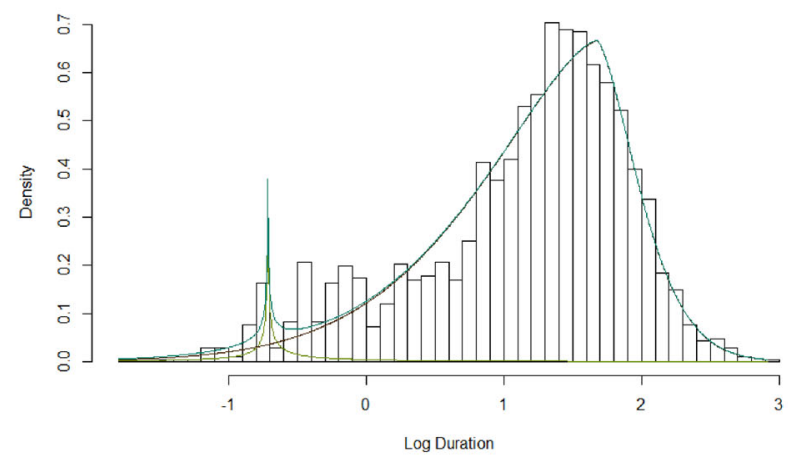

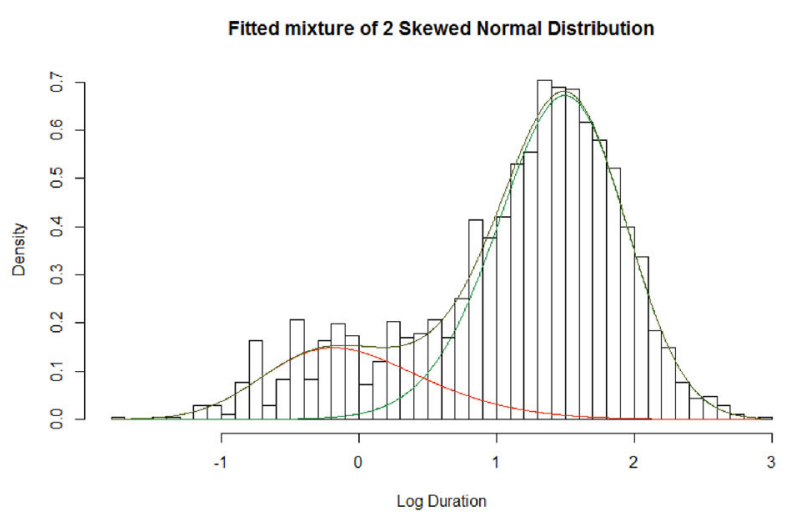

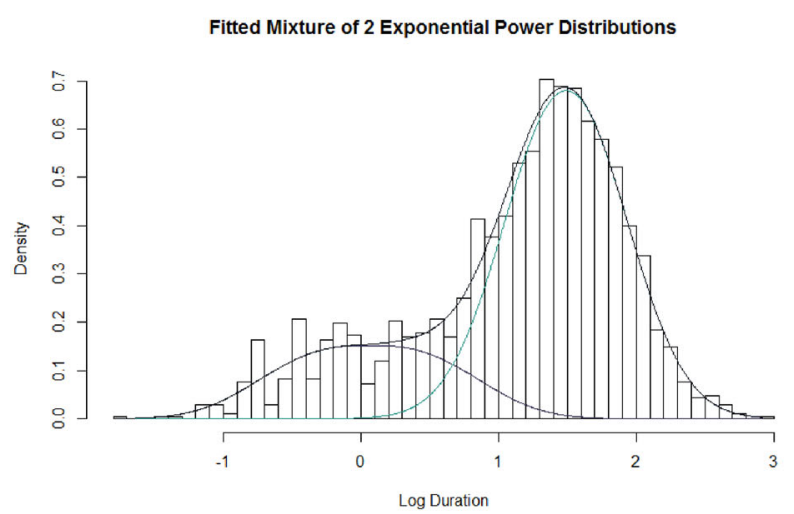

Figure 3. Density of the distributions fitted to the data set Fermi 2017.

the unusual fitting is caused by a large amount of ties existing in the lower portion of the data set, which subsequently results in an unnatural high peak having a high impact on the maximumlikelihood estimates. If we exclude the results of the two skew exponential power distribution, we see that the two skew normal distribution fits best in terms of all of the five information criteria with very strong evidence in terms of BIC and CAIC. The two exponential power distribution arguably fits as well as the two skew normal distribution, with only a 0.5 difference in all five criteria. One mixture component of the two skew normal distribution has positive skewness. The other has negative skewness. The component with negative skewness has the larger weight and smaller scale. It is located to the right-hand side of the other component.

\subsubsection{Fermi 2017}

There is very strong evidence $(\triangle \mathrm{BIC}, \triangle \mathrm{CAIC}>10)$ that both the two skew normal and two exponential power distributions are better models than the two skew exponential power distribution. Similar to the results in Section 5.1.2, unusual fits were recorded in the two skew exponential power distribution (see the density, QQ and PP plots). Excluding the two skew exponential power distribution, we have strong evidence that the two skew normal distribution fits best $(\triangle \mathrm{BIC}, \triangle \mathrm{CAIC}>6)$. Again, the two exponential power distribution performs very closely to the two skew normal distribution, with a 0.7 difference in all five criteria. Once again, one mixture component of the two skew normal distribution has positive skewness, and the other has negative skewness with the larger weight and smaller scale. 
Fitted Mixture of 3 Gaussian Distributions

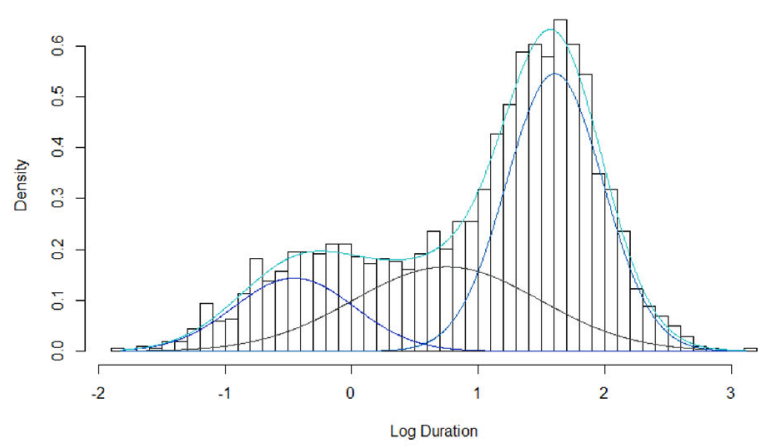

Fitted Mixture of 2 Generalized t Distributions

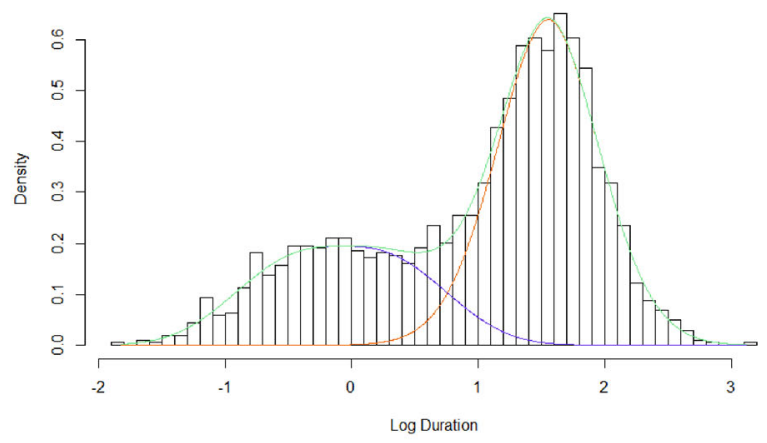

Fitted Mixture of 2 Skewed Exponential Power Distributions

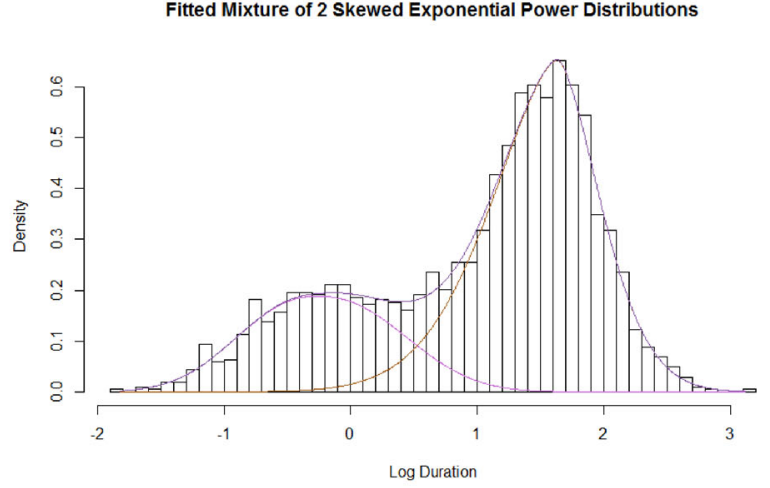

Fitted mixture of 2 Skewed Normal Distribution

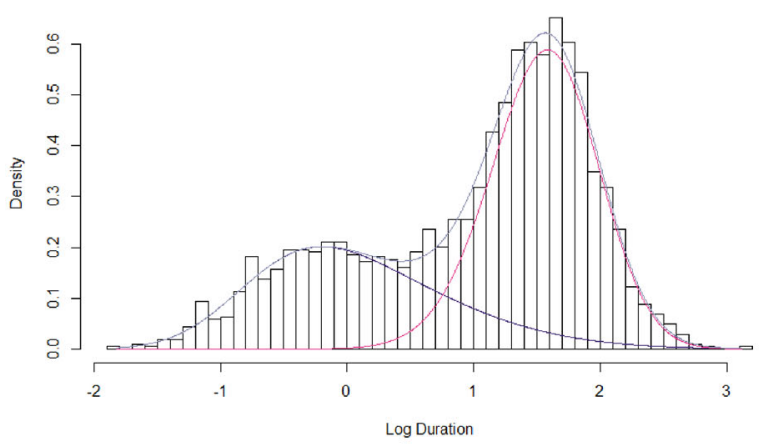

Fitted Mixture of 2 Exponential Power Distributions

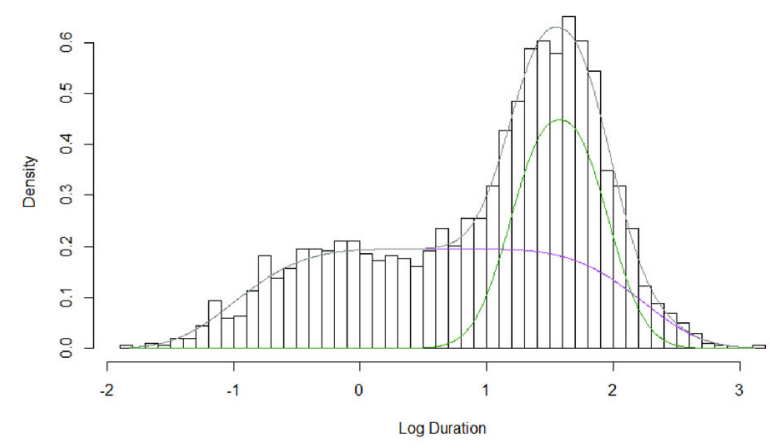

Figure 4. Density of the distributions fitted to the data set BATSE.

\section{$5.2 B A T S E$}

The two exponential power distribution performs best. With the lowest values in all of the five information criteria, there is strong evidence $(\triangle \mathrm{BIC}, \triangle \mathrm{CAIC}>7)$ that it is a better model than the three Gaussian, two generalized- $t$ and two skew exponential power distributions. There is also weak evidence $(\triangle \mathrm{AIC}>2 ; \triangle \mathrm{BIC}$, $\Delta$ CAIC > 2.2) that the two exponential power distribution fits better than the two skew normal distribution. All five distributions appear to give adequate fits according to the density, PP and QQ plots. Considering both the information criteria and plots, there is arguably enough evidence that the two exponential power distribution is the best distribution to describe this data set. Each mixture component of the two exponential power distribution has a kurtosis less than that of the Gaussian distribution, meaning each component has heavier tails. The component having the larger weight has the larger scale and smaller kurtosis. It is located to the left-hand side of the component having the smaller weight.

\subsection{Swift}

While both the two skew normal and two exponential power distributions performed the best in Sections 5.1.1, 5.1.2, 5.1.3 and 5.2, for this data set, there is very strong evidence $(\triangle \mathrm{AIC}>10)$ and strong evidence $(\triangle \mathrm{BIC}, \triangle \mathrm{CAIC}>7)$ to support the three Gaussian distribution against the two exponential power distribution. There is positive evidence that $(\triangle \mathrm{BIC}, \triangle \mathrm{CAIC}>4)$ the two skew normal distribution fits better than the three Gaussian distribution. The density, PP and QQ plots show that the three Gaussian distribution fits the data better than the other four distributions. The component of the three Gaussian distribution having the largest weight also has the largest scale and it is located in the middle of the two other 


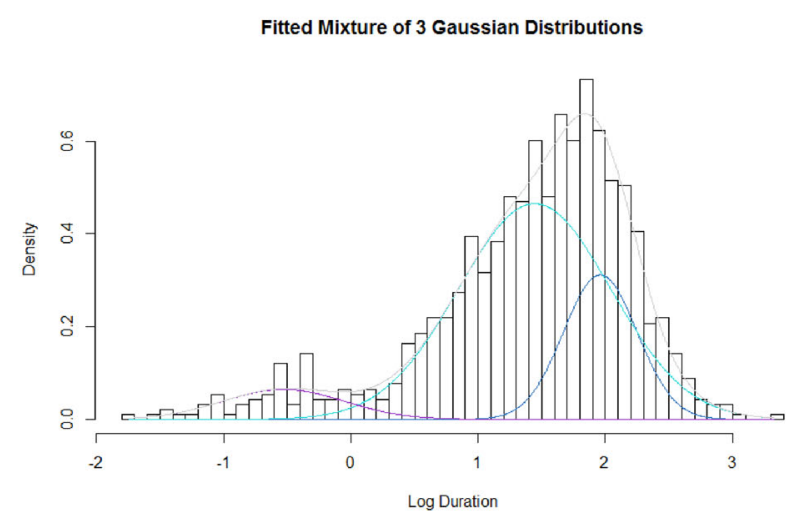

Fitted Mixture of 2 Generalized t Distributions

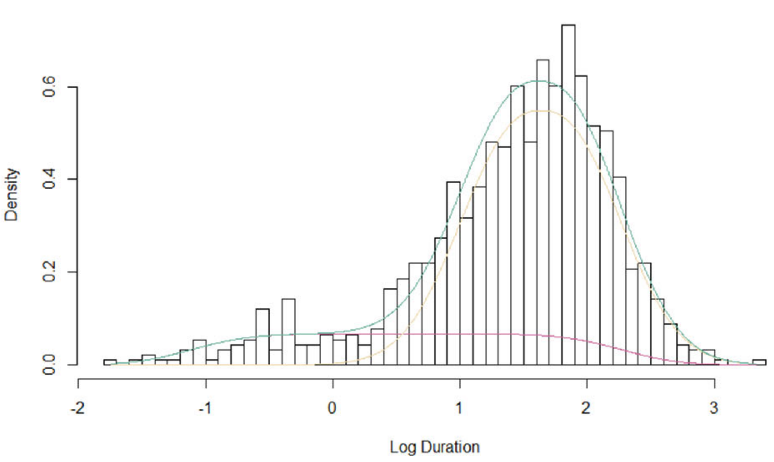

Fitted Mixture of 2 Skewed Exponential Power Distributions

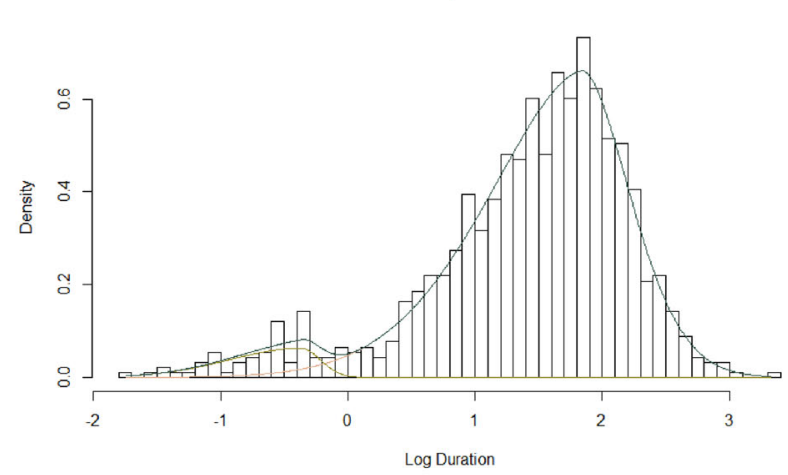

Fitted mixture of 2 Skewed Normal Distribution

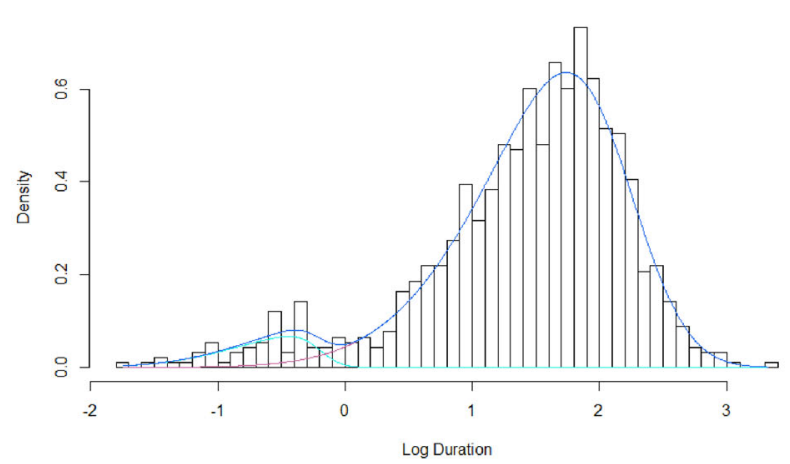

Fitted Mixture of 2 Exponential Power Distributions

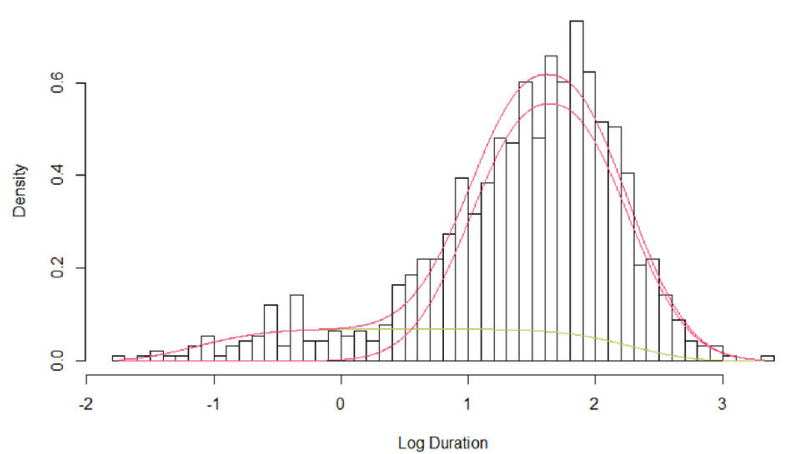

Figure 5. Density of the distributions fitted to the data set Swift.

components. The component having the second largest weight has the smallest scale, and it is located to the right-hand side of the two other components. The component having the smallest weight has the second smallest scale, and it is located to the left-hand side of the two other components.

A possible reason for the better fit of the three Gaussian distribution could be that this data set has less significant bimodality. Figs 1-5 show that the bimodality of $\ln T_{90}$ from Fermi and BATSE is more significant than that from Swift. Tables 4-12 show that the best-fitting exponential power distributions are a mixture of a platykurtic distribution and a slightly platykurtic distribution (close to a Gaussian distribution). A possible reason that the two exponential power distribution fits all previous data sets better than the three Gaussian distribution is its better fits to the lower half of each data set. For the data set Swift, the less significant bimodality makes the contribution of the fit of the exponential power distribution to the lower half of the data set less significant.

\subsection{Comparison of the three data bases}

For most data sets, the difference in log likelihoods between the best-fitting and three Gaussian distributions is small. The exception is the Fermi 2015 data set. The best-fitting distributions for Fermi 2015 without outliers, Fermi 2017 and BATSE, were chosen on the basis of the five information criteria: For the Fermi 2015 data without outliers, the differences between the best-fitting and three Gaussian distributions are substantial for all five criteria; for the Fermi 2017 data, the differences between the best-fitting and three Gaussian distributions are substantial for BIC, CAIC and HQC; and for the BATSE data, the differences between the best-fitting 
QQ-plot: Fitted mixture of 3 Gaussian Distributions

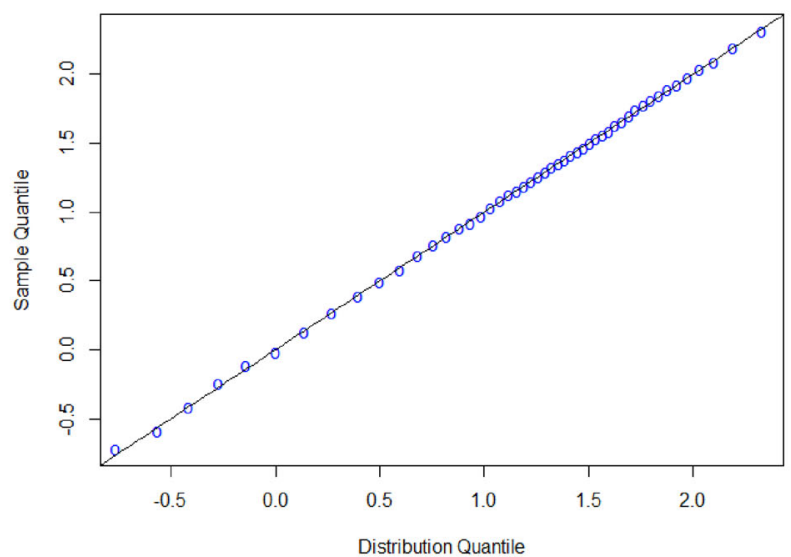

QQ-plot: Fitted mixture of 2 Generalized t Distributions

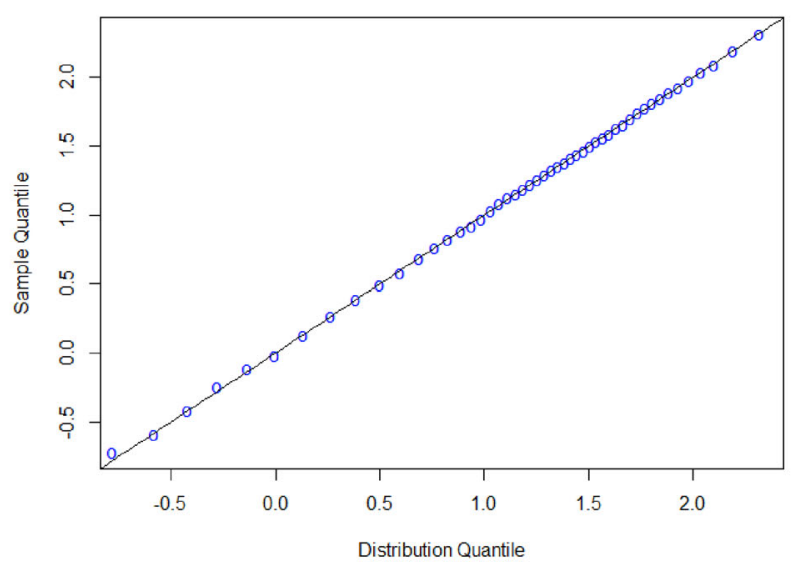

QQ-plot: Fitted mixture of 2 Skewed exponential power Distributions

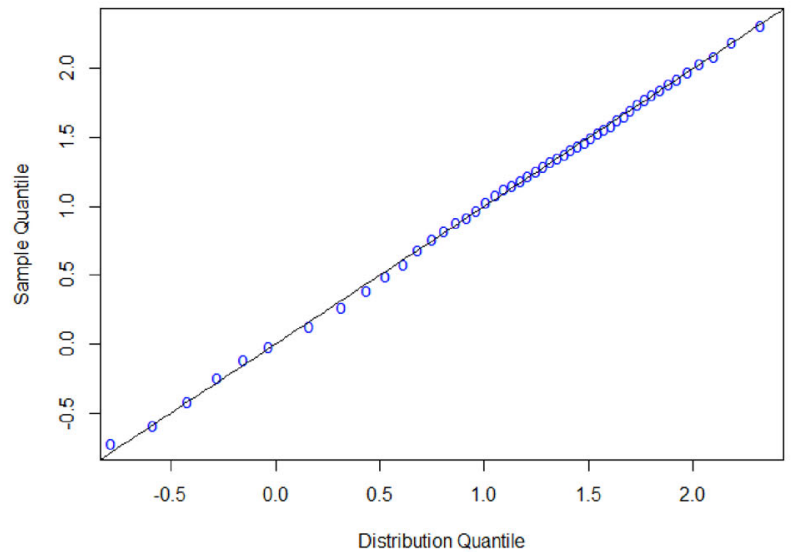

QQ-plot: Fitted mixture of 2 Skewed Normal Distribution

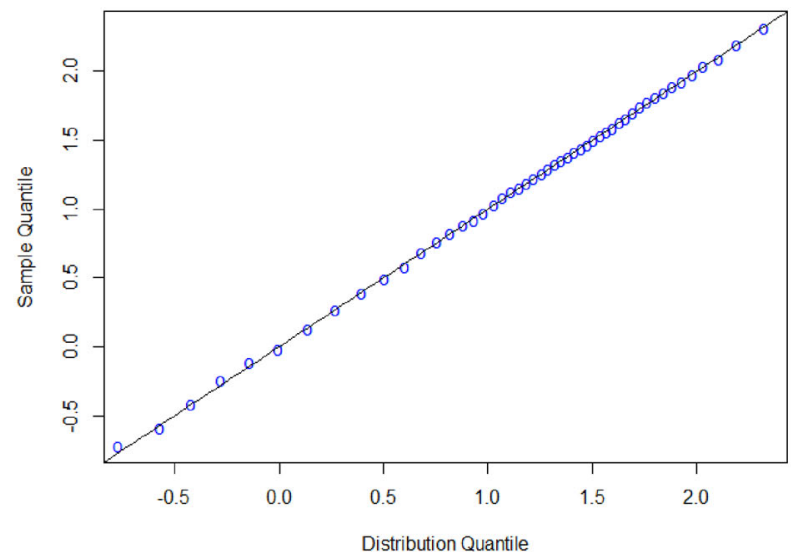

QQ-plot: Fitted mixture of 2 Exponential power Distributions

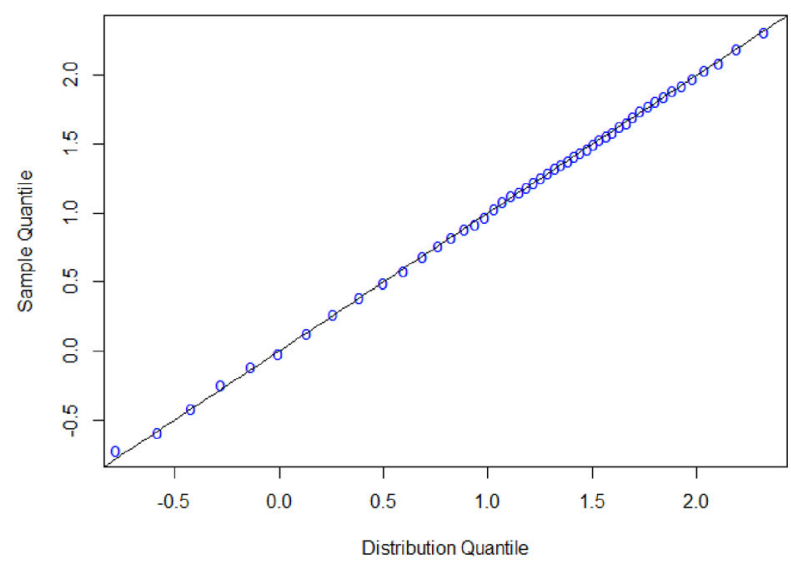

Figure 6. QQ-plot of the distributions fitted to the data set Fermi 2015 with outliers excluded.

and three Gaussian distributions are substantial for BIC, CAIC and HQC.

\section{CONCLUSIONS}

Both two skew normal and two exponential power distributions provide significantly better fits than the three Gaussian distribution for the gamma-ray burst durations recorded by Fermi and BATSE. The two distributions exhibit distinct properties: The extra parameter in the skew normal distribution adds different levels of skewness to the Gaussian distribution, and the extra parameter in the exponential power distribution adds different levels of kurtosis to the Gaussian distribution. The exponential power distribution has an elementary expression for its probability density function, which 
QQ-plot: Fitted mixture of 3 Gaussian Distributions

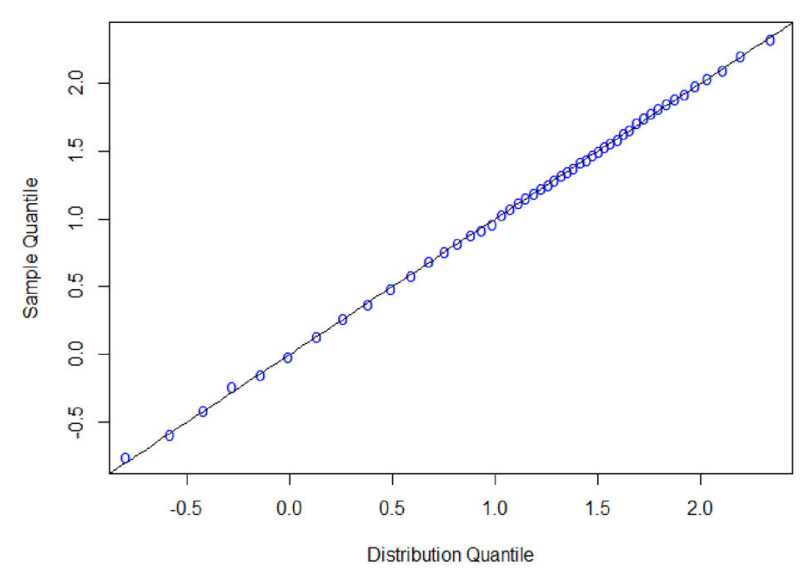

QQ-plot: Fitted mixture of 2 Generalized t Distributions

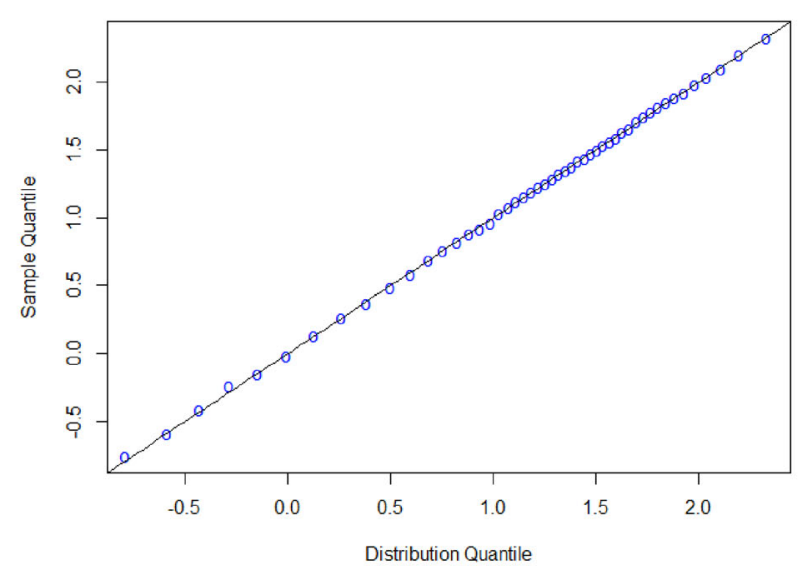

QQ-plot: Fitted mixture of 2 Skewed exponential power Distributions

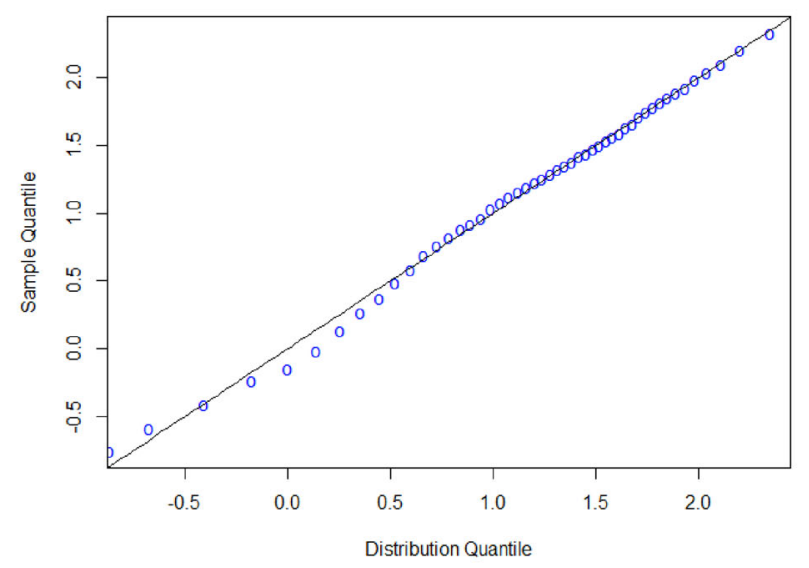

QQ-plot: Fitted mixture of 2 Skewed Normal Distribution

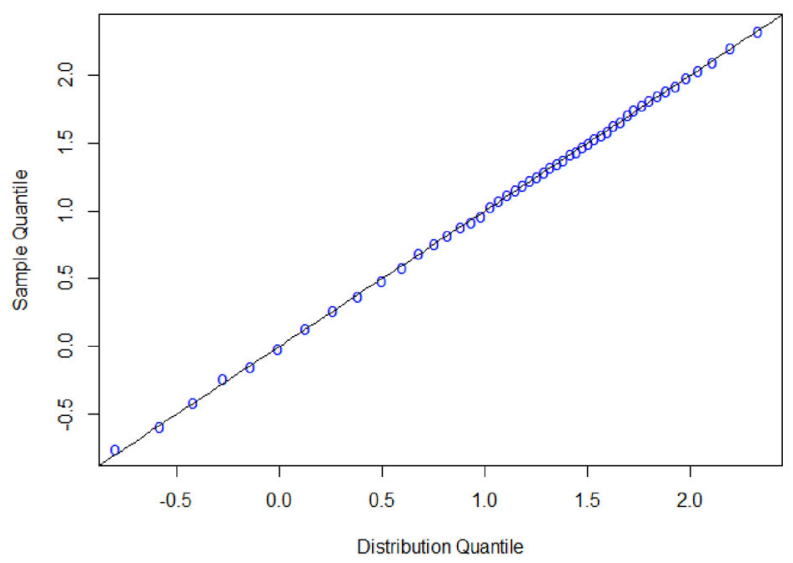

QQ-plot: Fitted mixture of 2 Exponential power Distributions

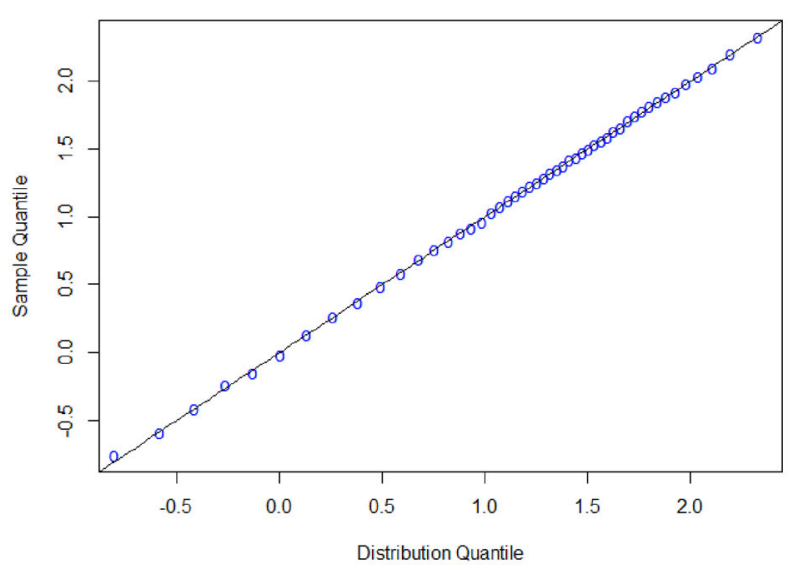

Figure 7. QQ-plot of the distributions fitted to the data set Fermi 2015.

is not the case for the skew normal distribution. The exponential power distribution also has a closed-form expression for its cumulative distribution function. The skew normal distribution does not have a closed-form cumulative distribution function. Hence, the exponential power distribution should be preferred for practical use.

The number of different groups of gamma-ray burst durations is more likely to be two than three. However, we can-not find statistical evidence to tell whether gamma-ray burst durations have a skew distribution or a platykurtic distribution as they both fit equally well. We also believe that the high amount of ties in Fermi could have affected the skewness and kurtosis, especially when the data are in logarithmic scale.

For the Swift data, although the two skew normal distribution fits better than the three Gaussian distribution in terms of BIC 
QQ-plot: Fitted mixture of 3 Gaussian Distributions

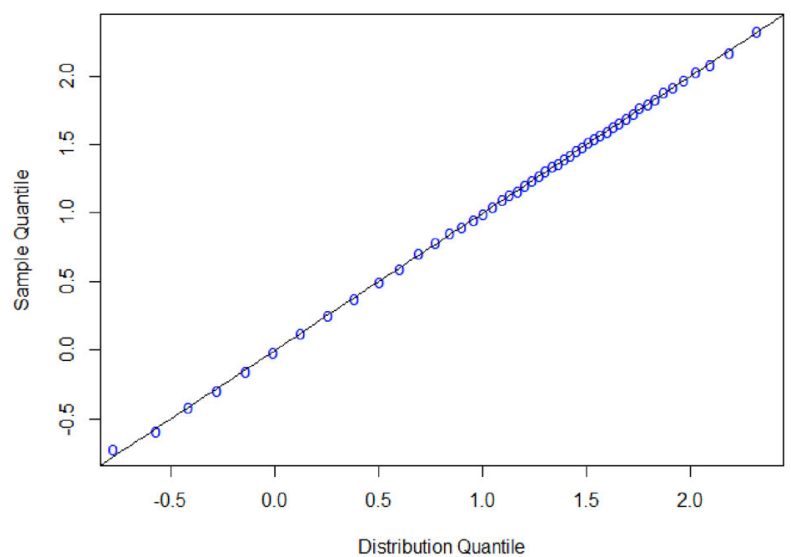

QQ-plot: Fitted mixture of 2 Generalized t Distributions

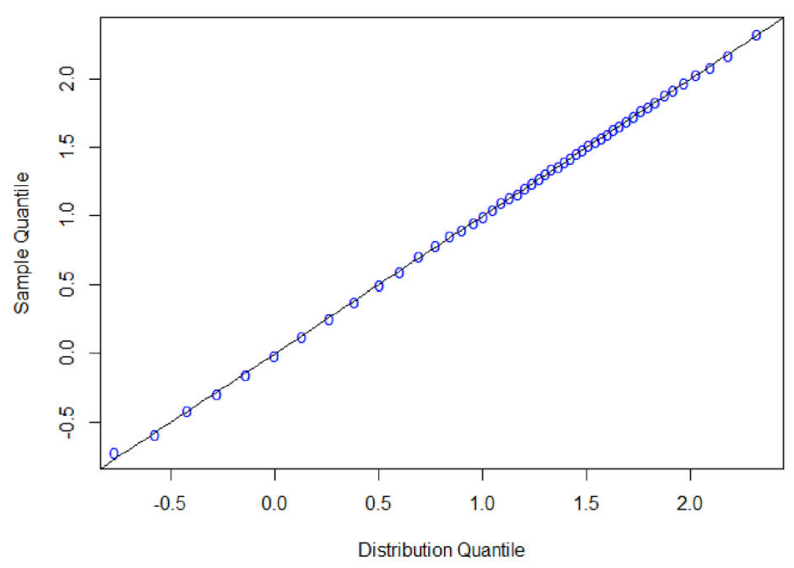

QQ-plot: Fitted mixture of 2 Skewed exponential power Distributions

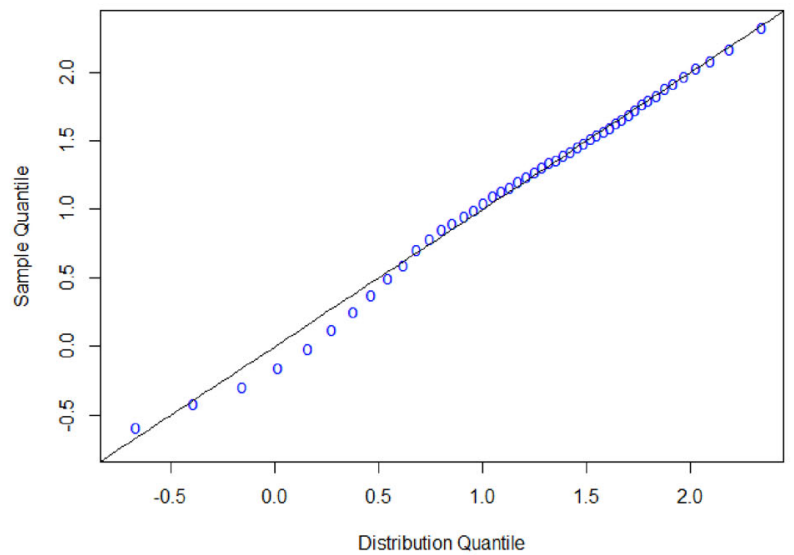

QQ-plot: Fitted mixture of 2 Skewed Normal Distribution

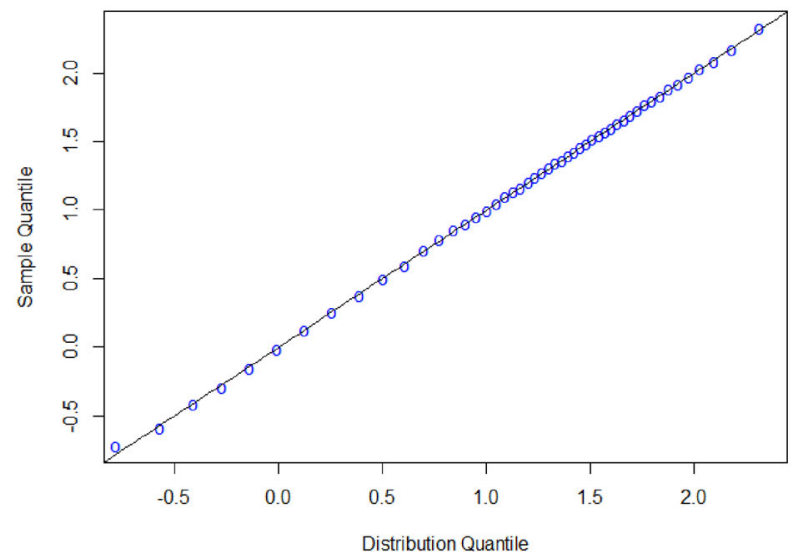

QQ-plot: Fitted mixture of 2 Exponential power Distributions

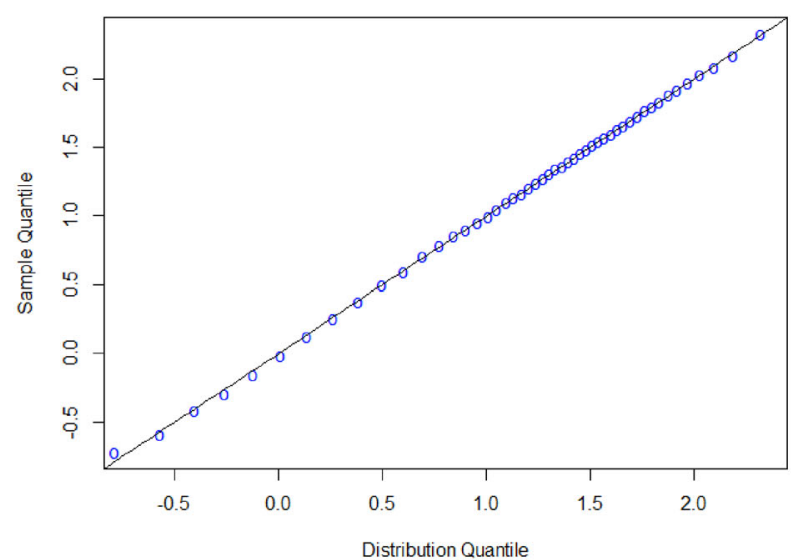

Figure 8. QQ-plot of the distributions fitted to the data set Fermi 2017.

and CAIC, the three Gaussian distribution provides the best fit in terms of QQ-plot, PP-plot and other criteria. While we cannot formally find reasons why both two skew normal and two exponential power distributions perform worse, we believe that reasons could be due to differences in gamma-ray burst detector sensitivities (Band 2006) and the existence of ties. Moreover, the fitted parameters of the three Gaussian distribution are significantly different from those for other data sets. For example, the third group of the three Gaussian distribution has a significantly higher mean and proportion compared to other data sets. This suggests that the third group in Swift may be closely related to the sensitivity of the Swift detector. An astrophysical meaning for the 
QQ-plot: Fitted mixture of 3 Gaussian Distributions

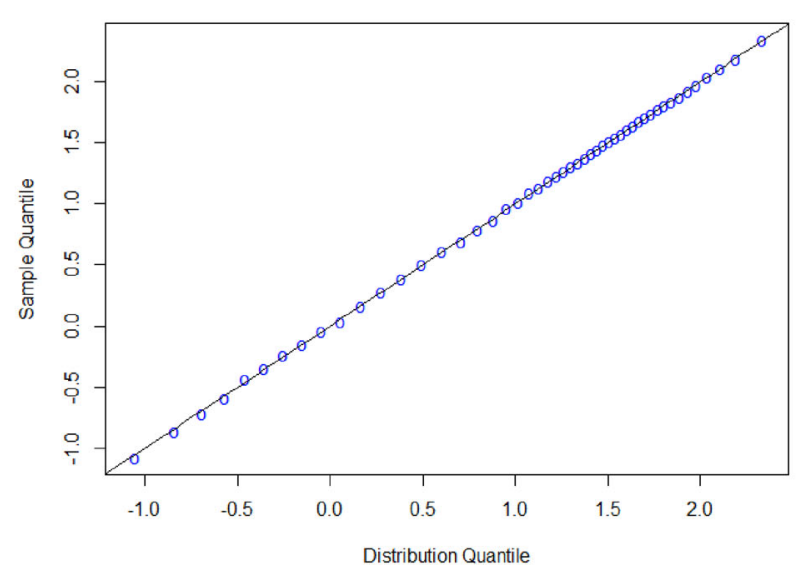

QQ-plot: Fitted mixture of 2 Generalized t Distributions

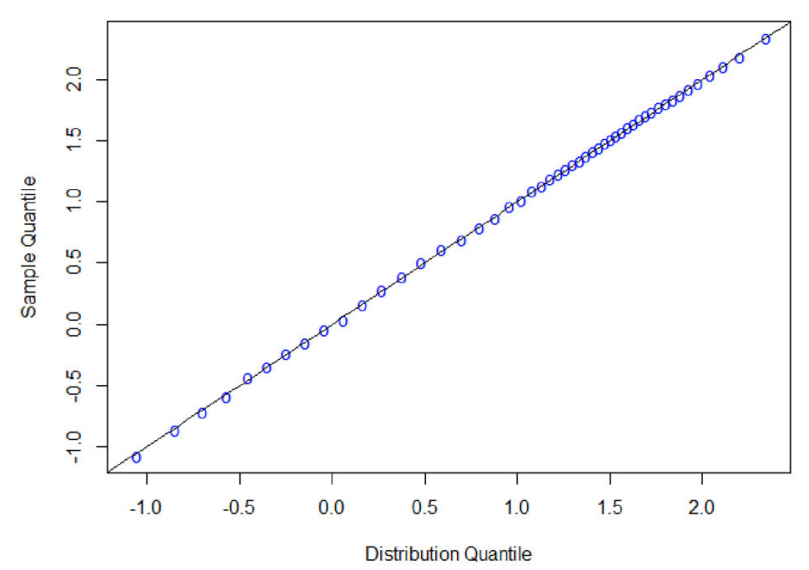

QQ-plot: Fitted mixture of 2 Skewed exponential power Distributions

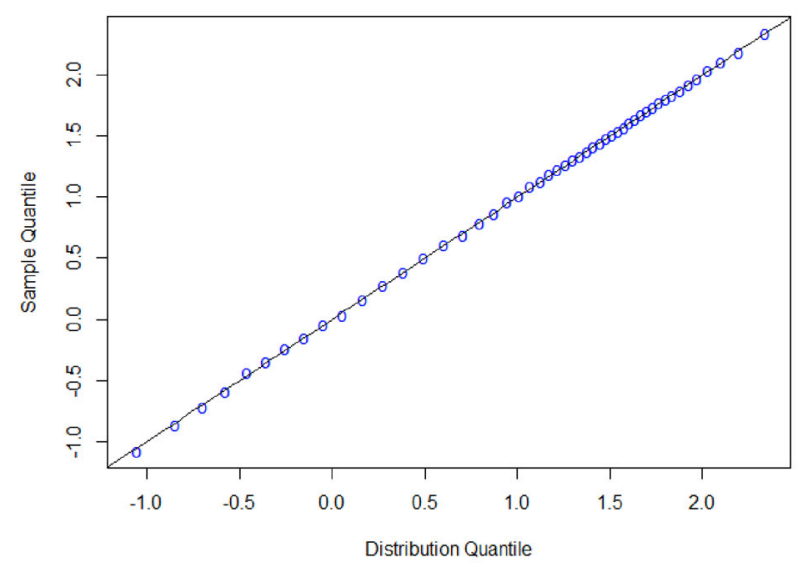

QQ-plot: Fitted mixture of 2 Skewed Normal Distribution

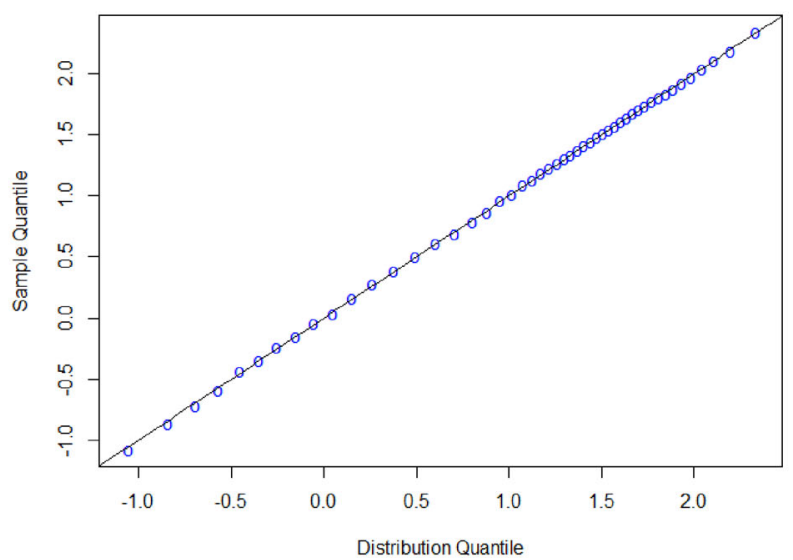

QQ-plot: Fitted mixture of 2 Exponential power Distributions

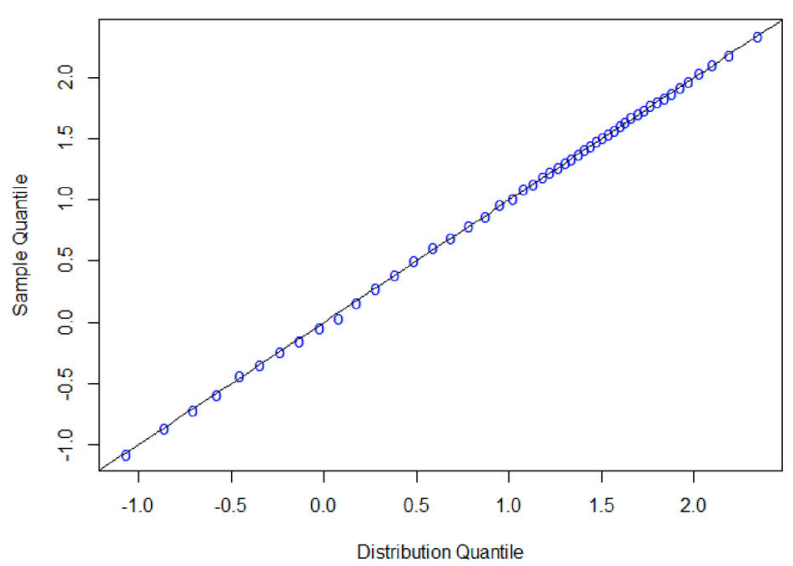

Figure 9. QQ-plot of the distributions fitted to the data set BATSE.

presence of the third group is presented in Veres et al. (2010). They found evidence that the third group 'is closely related to X-ray flashes'.

The best-fitting distributions in Section 5 compared to those in Tarnopolski (2016) are as follows: For the Fermi 2015 data with outliers excluded, the two exponential power distribution provides better fits than the two skew normal distribution of Tarnopolski (2016) in terms of each of the five criteria, although the differences are small; and for the BATSE data, the two exponential power distribution provides better fits than the three Gaussian distribution of Tarnopolski (2016) in terms of each of the five criteria; most of the differences suggest either positive or strong evidence. Hence, the 
QQ-plot: Fitted mixture of 3 Gaussian Distributions

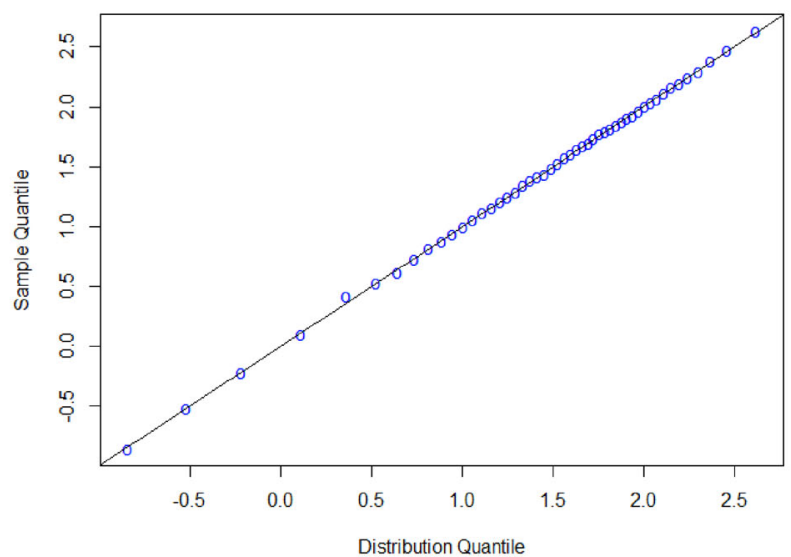

QQ-plot: Fitted mixture of 2 Generalized t Distributions

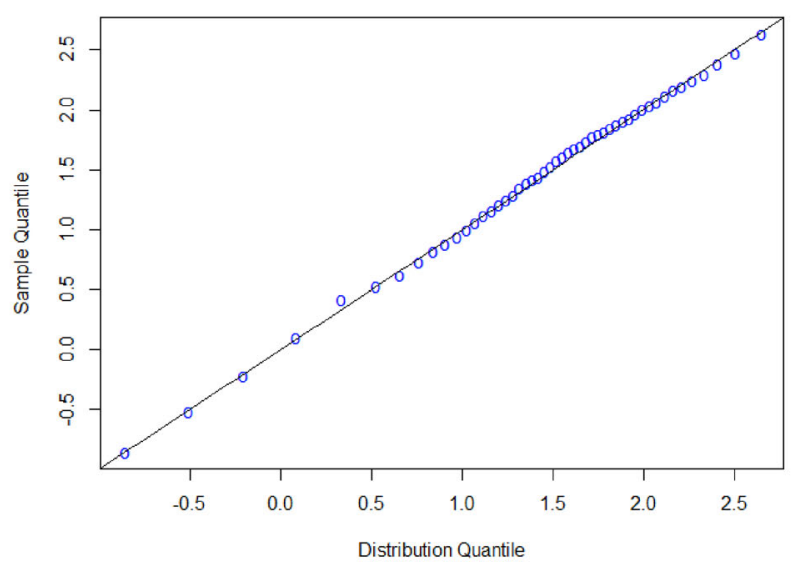

QQ-plot: Fitted mixture of 2 Skewed exponential power Distributions

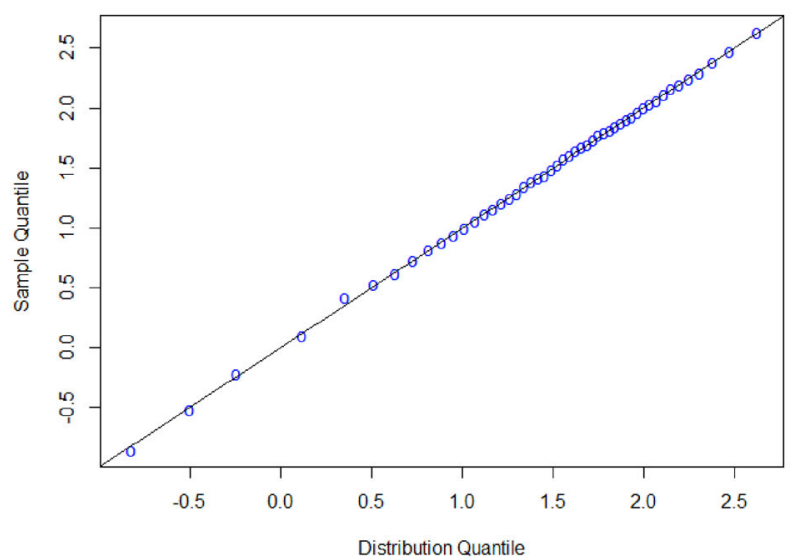

QQ-plot: Fitted mixture of 2 Skewed Normal Distribution

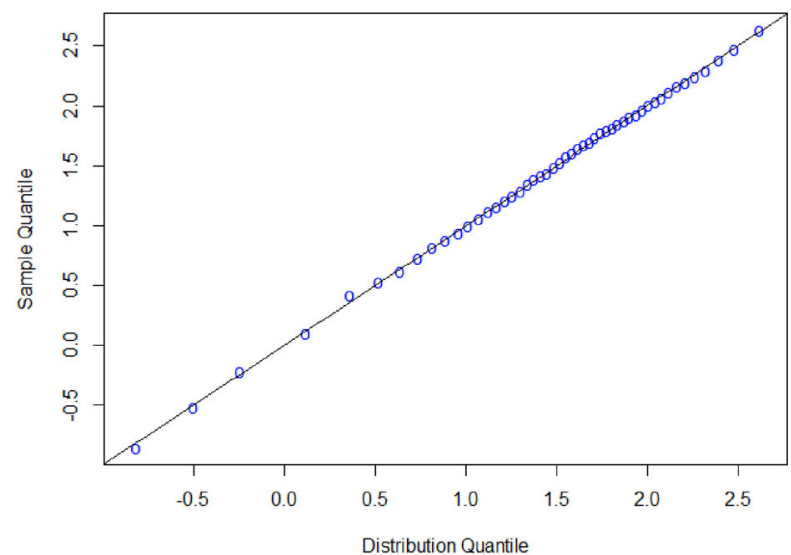

QQ-plot: Fitted mixture of 2 Exponential power Distributions

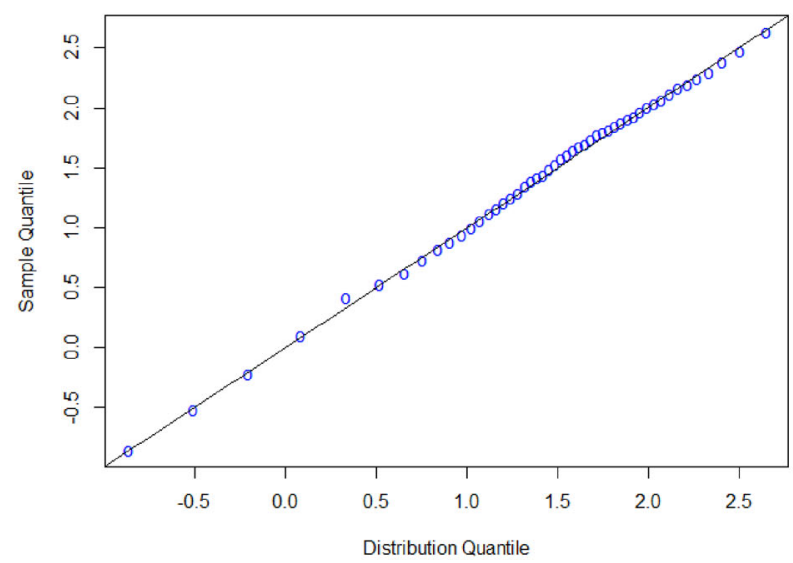

Figure 10. QQ-plot of the distributions fitted to the data set Swift.

two exponential power distribution has gains over those discussed in Tarnopolski (2016).

The three Gaussian distribution giving the best fit can be attributed to the Central Limit Theorem (Durrett 2004). We also have two other distributions giving best fits for $T_{90}$ durations. The two skew normal distribution giving the best fit can be attributed to varying degrees of skewness in the data: One part of the data has positive skewness, while the other part has negative skewness, as shown in Section 5.1.2 for the Fermi 2015 data set and Section 5.1.3 for the Fermi 2017 data set. The two exponential power distribution giving the best fit can be attributed to the presence of heavier tails than the Gaussian distribution. It is also attributable to different degrees of 
PP-plot: Fitted mixture of 3 Gaussian Distributions

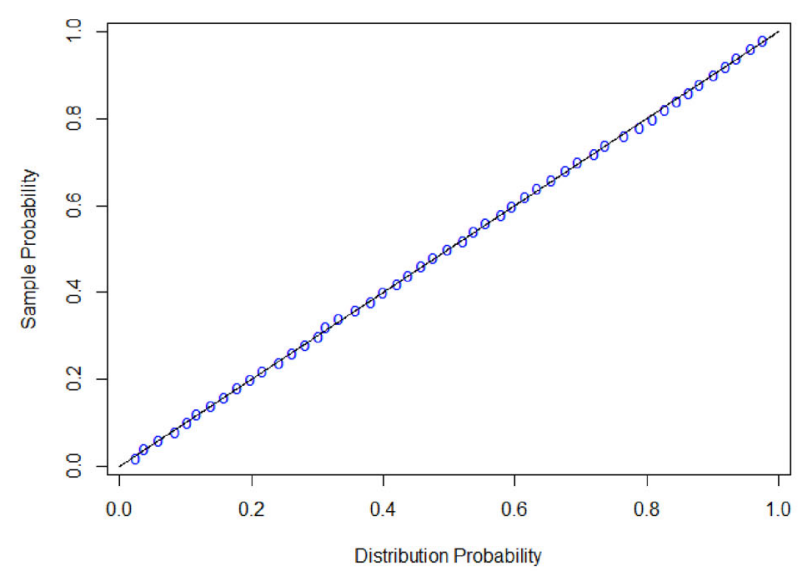

PP-plot: Fitted mixture of 2 Generalized t Distributions

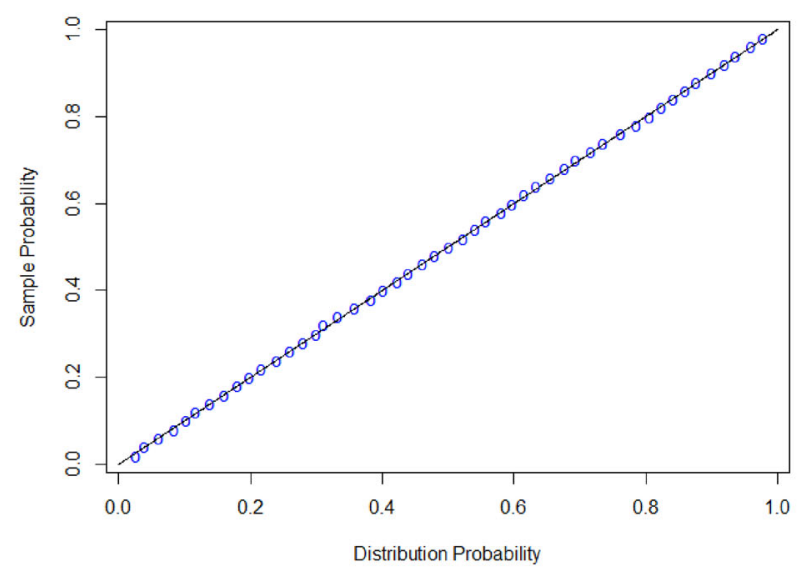

PP-plot: Fitted mixture of 2 Skewed exponential power Distributions

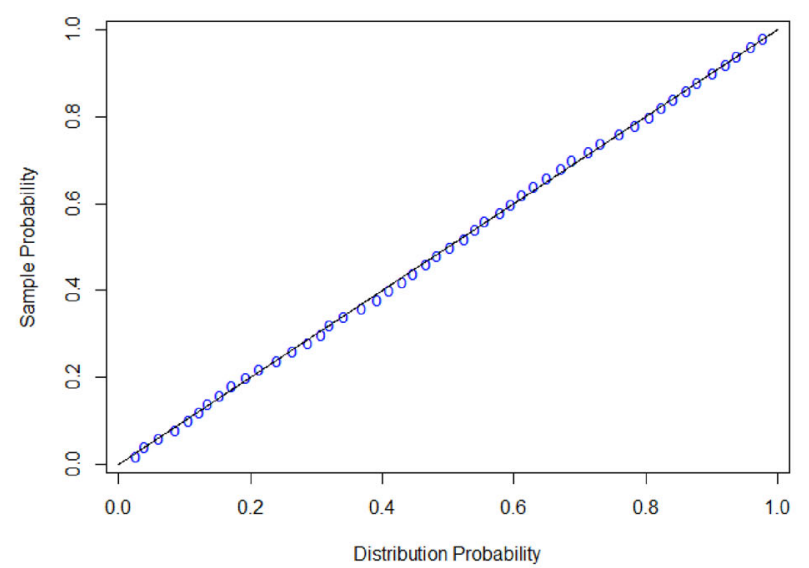

PP-plot: Fitted mixture of 2 Skewed Normal Distribution

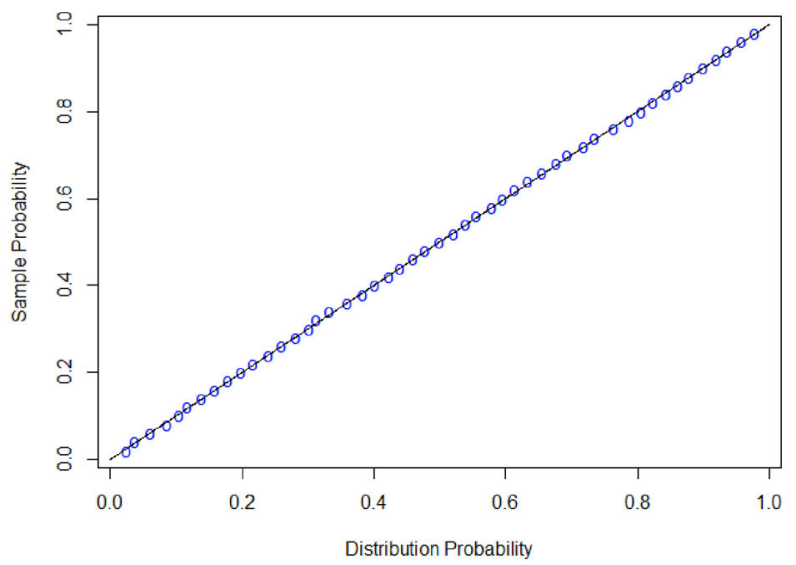

PP-plot: Fitted mixture of 2 Exponential power Distributions

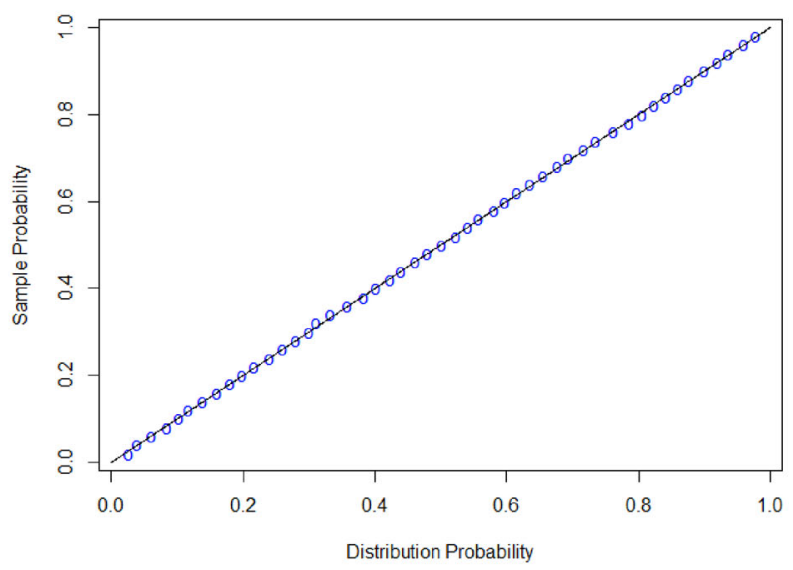

Figure 11. PP-plot of the distributions fitted to the data set Fermi 2015 with outliers excluded.

heavyness of the tails, one part of the data having heavy tails and the other part having even heavier tails, as shown in Section 5.1.1 for the Fermi 2015 data set with outliers excluded and Section 5.2 for the BATSE data set.

The message of this paper and Tarnopolski's $(2015,2016)$ is that often non-Gaussian distributions can provide better fits to gammaray burst durations. These include two skew normal, two sinh arc- sinh and two exponential power distributions. These distributions have fewer parameters than the three Gaussian distribution and hence are more efficient. Are there astrophysical explanations for the presence of non-Gaussianity in gamma-ray burst durations? This is an important question for future research. The three Gaussian distribution, however, appears to provide the best fit for the Swift data. The presence of the third group in this case may be due to 
PP-plot: Fitted mixture of 3 Gaussian Distributions

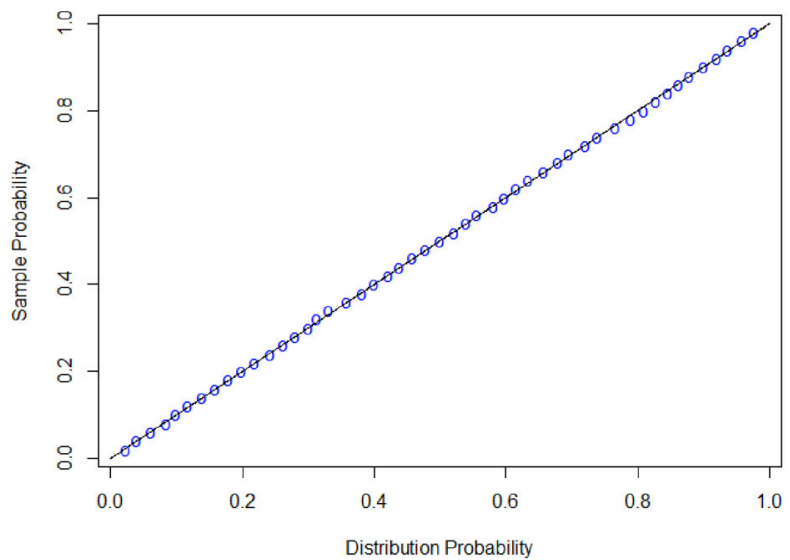

PP-plot: Fitted mixture of 2 Generalized t Distributions

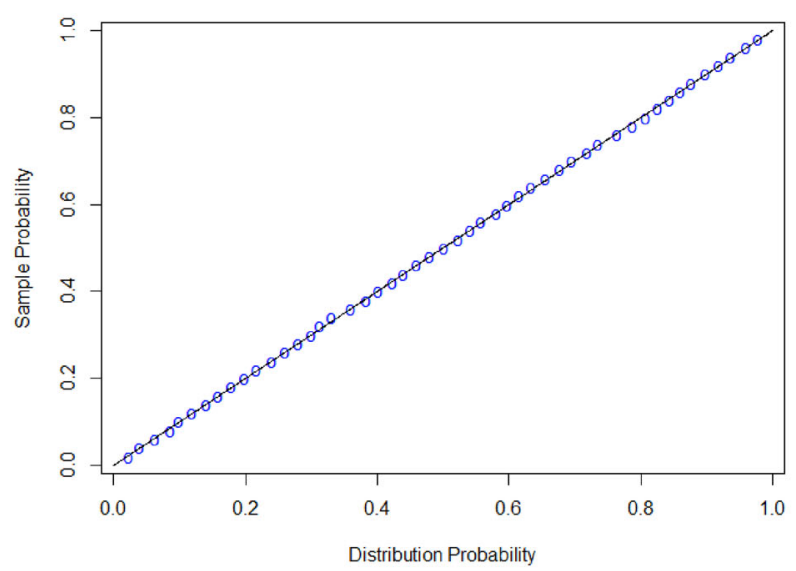

PP-plot: Fitted mixture of 2 Skewed exponential power Distributions

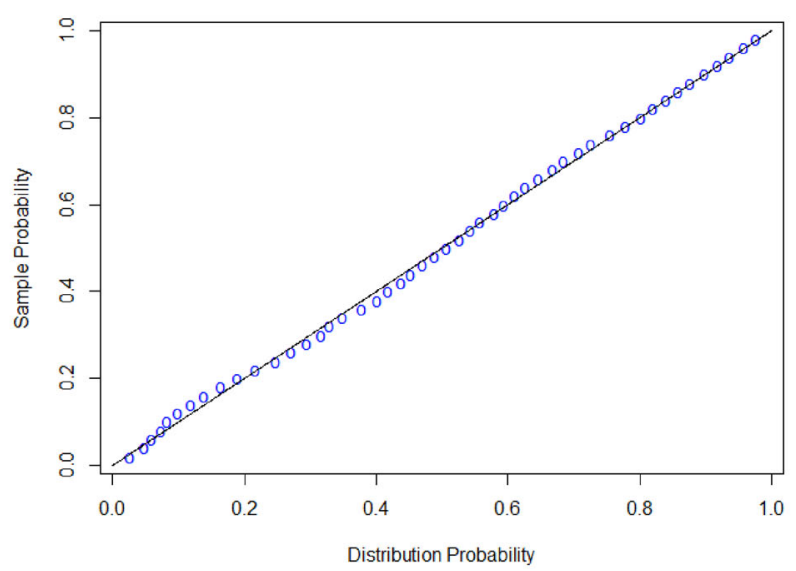

PP-plot: Fitted mixture of 2 Skewed Normal Distribution

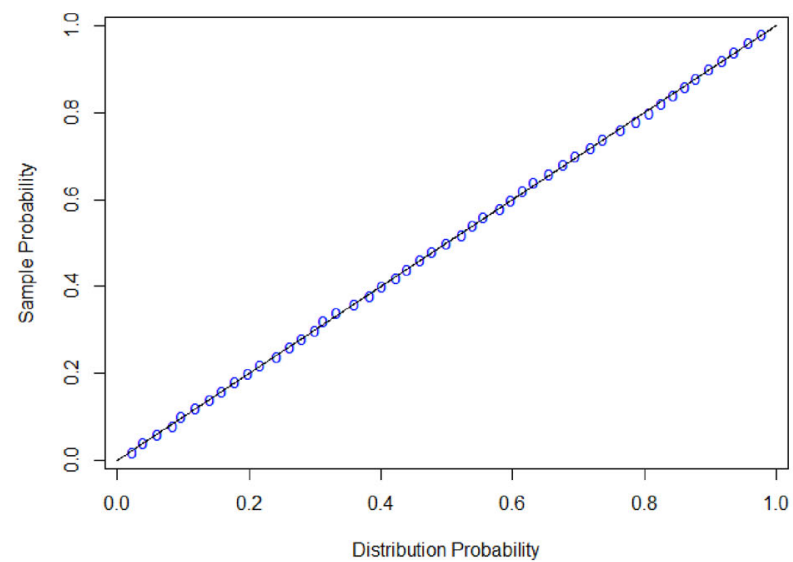

PP-plot: Fitted mixture of 2 Exponential power Distributions

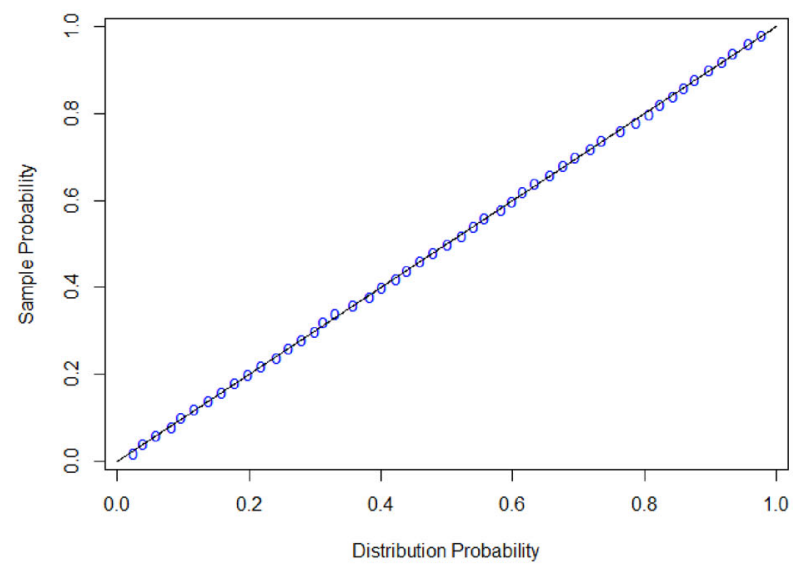

Figure 12. PP-plot of the distributions fitted to the data set Fermi 2015.

X-ray flashes, as explained in Veres et al. (2010). It is also possible that the presence of a third group may be just a characteristic of the Gaussian distribution. The third group might not have appeared if the Swift data had been fitted adequately by a non-Gaussian distribution.
Future works will include: (i) fitting non-parametric and semiparameter distributions to the data sets; ii) using gamma-ray burst data (for example, $T_{50}$ durations) having more dimensions; and (iii) performing classification analysis between different groups of gamma-ray burst durations. 

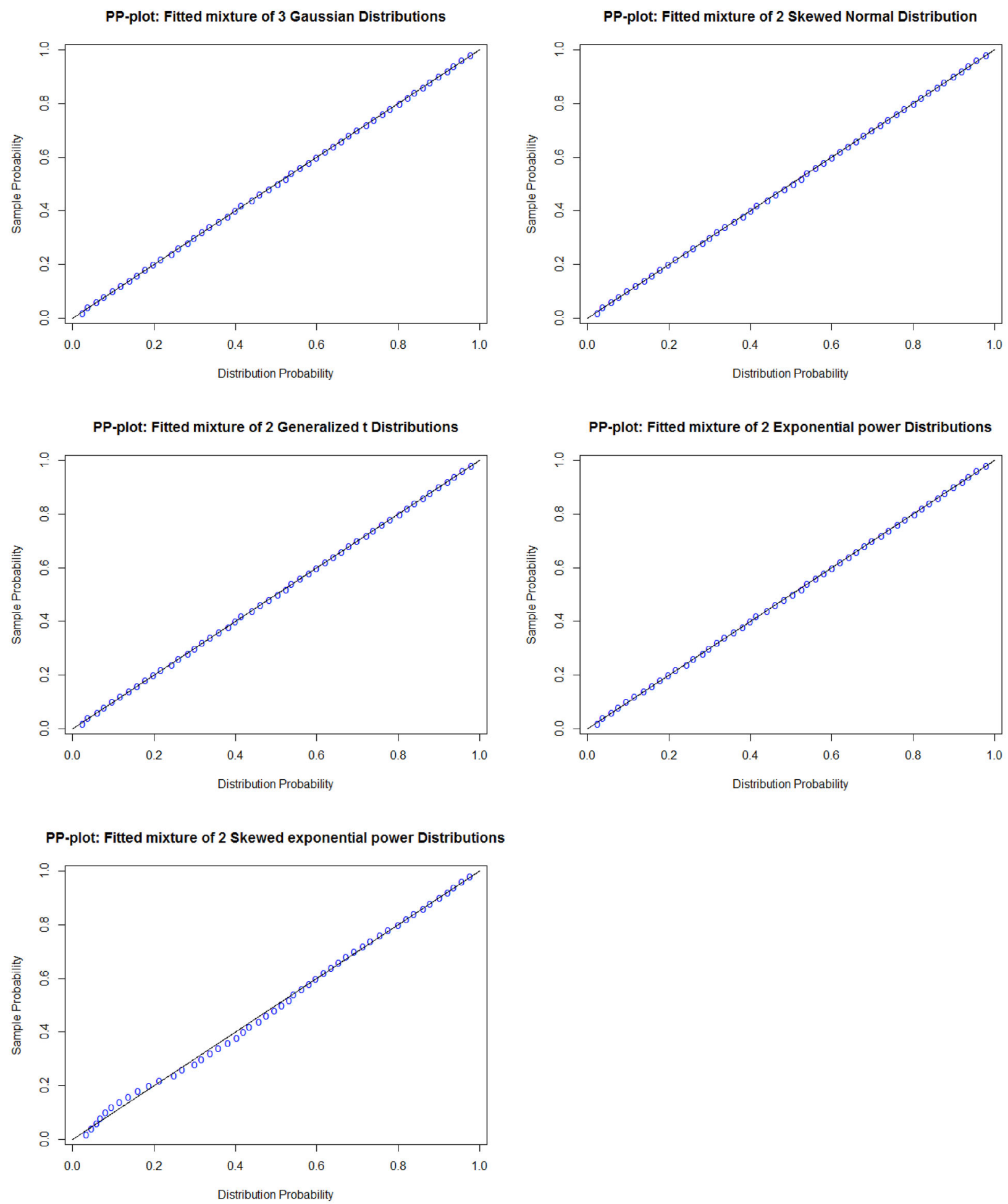

Figure 13. PP-plot of the distributions fitted to the data set Fermi 2017. 
PP-plot: Fitted mixture of 3 Gaussian Distributions

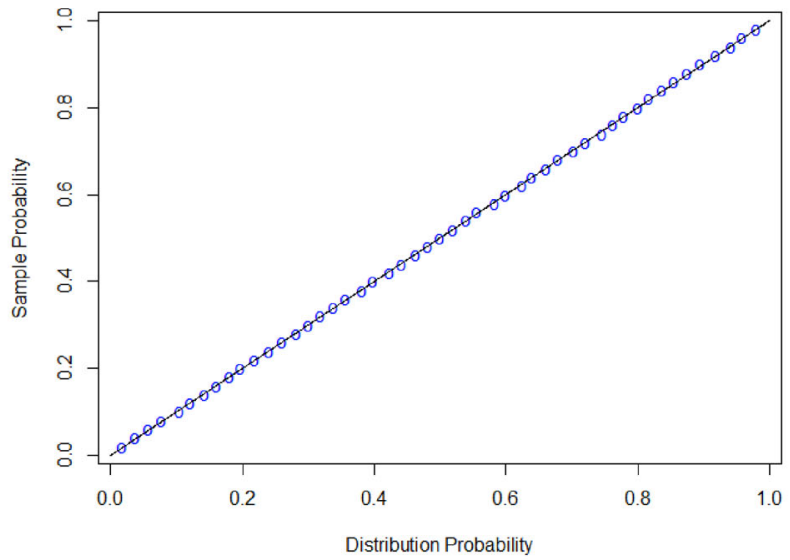

PP-plot: Fitted mixture of 2 Generalized t Distributions

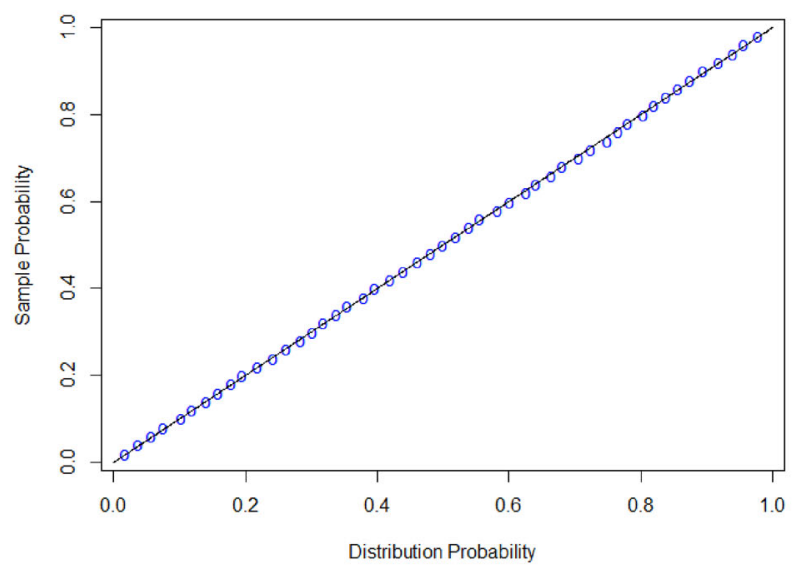

PP-plot: Fitted mixture of 2 Skewed exponential power Distributions

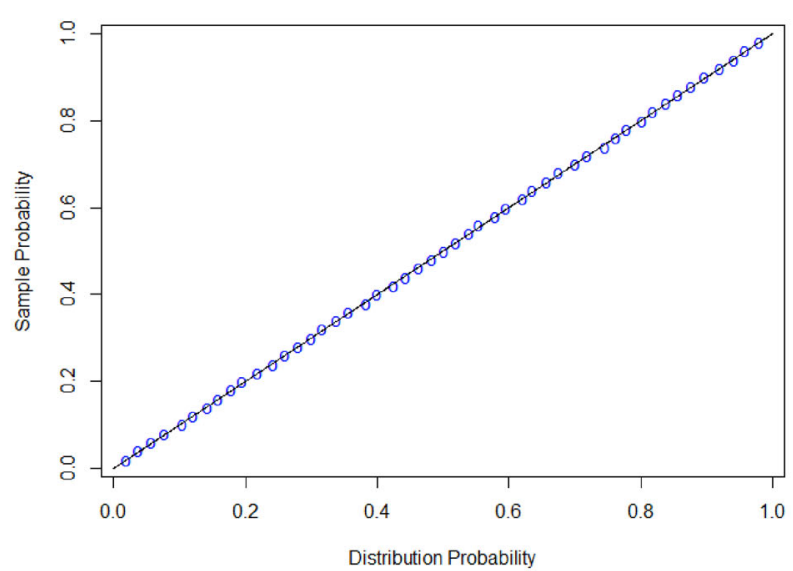

PP-plot: Fitted mixture of 2 Skewed Normal Distribution

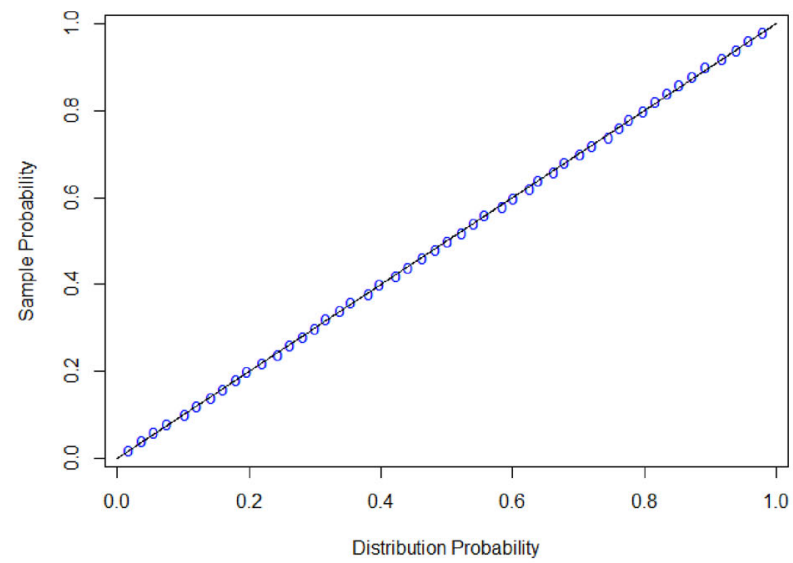

PP-plot: Fitted mixture of 2 Exponential power Distributions

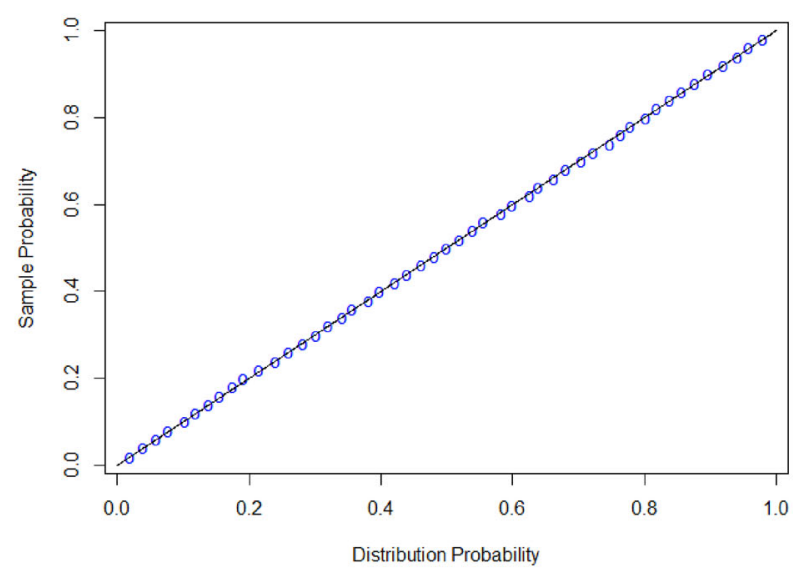

Figure 14. PP-plot of the distributions fitted to the data set BATSE. 
PP-plot: Fitted mixture of 3 Gaussian Distributions

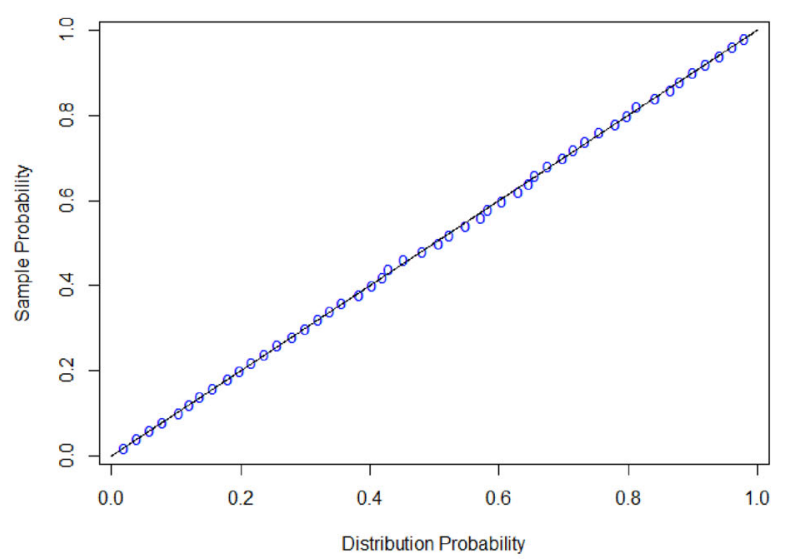

PP-plot: Fitted mixture of 2 Generalized t Distributions

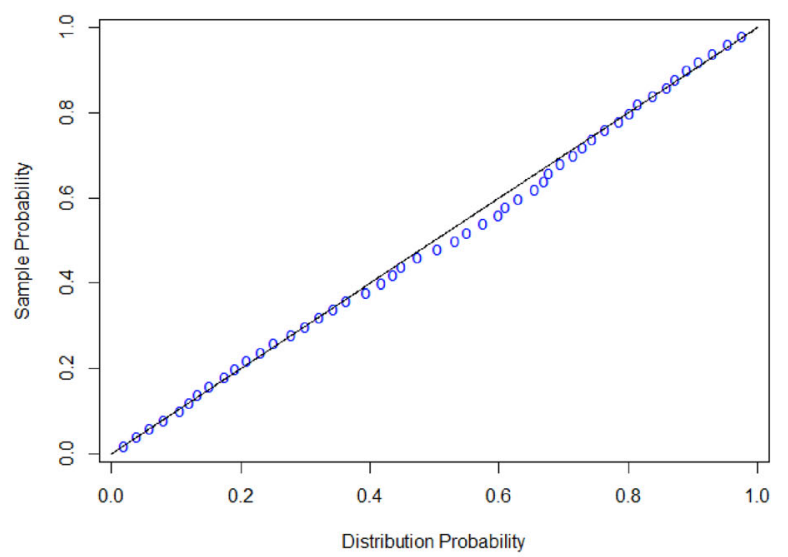

PP-plot: Fitted mixture of 2 Skewed exponential power Distributions

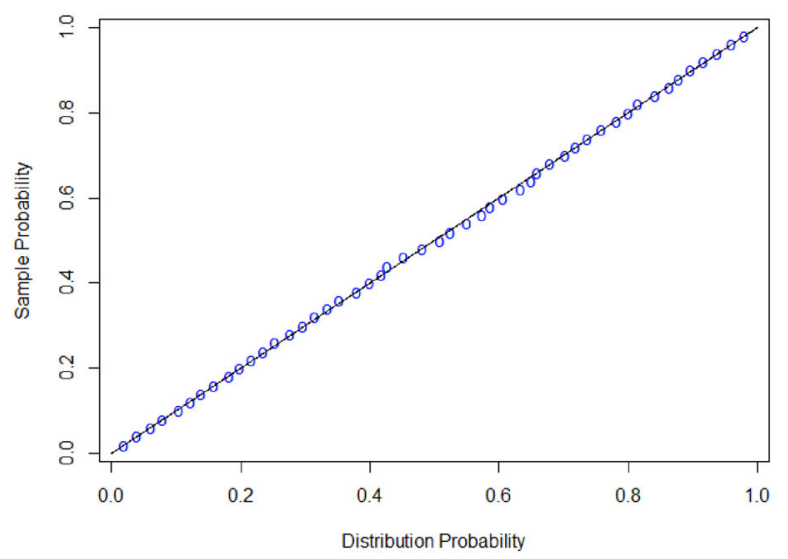

PP-plot: Fitted mixture of 2 Skewed Normal Distribution

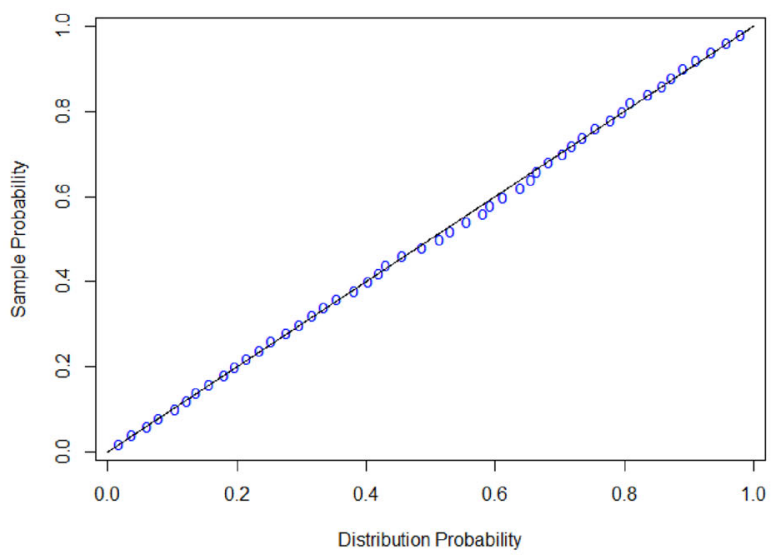

PP-plot: Fitted mixture of 2 Exponential power Distributions

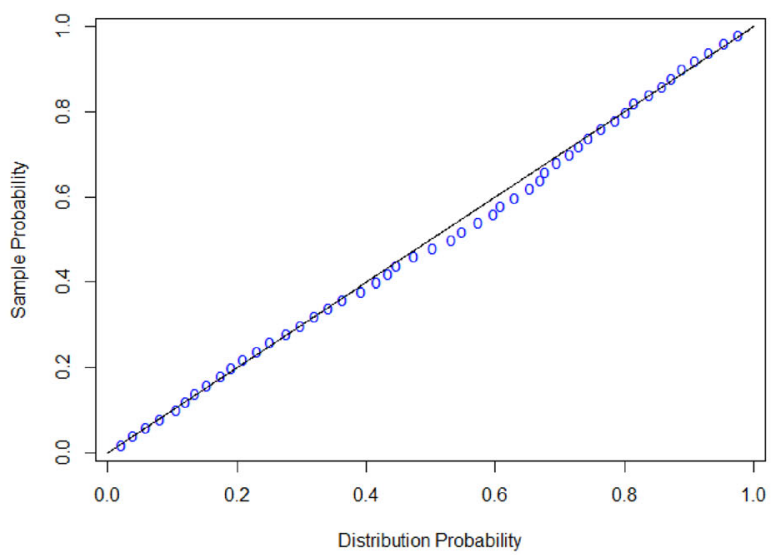

Figure 15. PP-plot of the distributions fitted to the data set Swift.

\section{ACKNOWLEDGEMENTS}

The authors would like to thank the referee and the editor for careful reading and comments, which significantly improved this paper.

\section{REFERENCES}

Akaike H., 1974, IEEE Trans. Autom. Control 19, 716

Azzalini A., 1985, Scand. J. Stat., 12, 171
Band D. L., 2006, ApJ, 644, 378

Bhave A., Kulkarni S., Desai S., Srijith P. K., 2017, preprint (arXiv:1708.05668)

Bozdogan H., 1987, Psychometrika, 52, 345

Burnham K. P., Anderson D. R., 2004, Sociological Methods and Research, 33,261

Cox D. R., Hinkley D. V., 1974, Theoretical Statistics. Chapman and Hall, London

Dempster A. P., Laird N. M., Rubin D. B., 1977, J. R. Stat. Soc. B, 39, 1 
Durrett R., 2004, Probability: Theory and Examples, 3rd edn. Cambridge Univ. Press, New York

Fang Y., 2011, J. Data Sci., 9, 15

Hannan E. J., Quinn B. G., 1979, J. R. Stat. Soc. B, 41, 190

Horvath I., Balazs L. G., Bagoly Z., Veres P., 2008, A\&A, 489, L1

Horvath I., Bagoly Z., Balazs L. G., de Ugarte Postigo A., Veres P., Meszaros A., 2010, ApJ, 713, 552

Huja D., Meszaros A., Ripa J., 2009, A\&A, 504, 67

Hurvich C. M., Tsai C.-L., 1989, Biometrika, 76, 297

McDonald J. B., Newey W. K., 1988, Econometric Theory, 4, 428

Mukherjee S., Feigelson E. D., Babu U. J., Murtagh F., Fraley C., Raftery A., 1998, ApJ, 508, 314

R Development Core Team 2017, R: A Language and Environment for Statistical Computing. R Foundation for Statistical Computing. Vienna Raftery A. E., 1996, Biometrika, 83, 251

Ripa J., Meszaros A., 2016, Ap\&SS, 361, 1
Ripa J., Meszaros A., Veres P., Park I. H., 2012, ApJ, 756, 44

Savchenko V., Neronov A., Courvoisier T. J.-L., 2012, A\&A, 541, A122

Schwarz G. E., 1978, Ann. Stat., 6, 461

Subbotin M. T., 1923, Sbornik, 31, 296

Tarnopolski M., 2015, A\&A, 581, A29

Tarnopolski M., 2016, MNRAS, 458, 2024

Veres P., Bagoly Z., Horvath I., Meszaros A., Balazs L. G., 2010, ApJ, 725, 1955

Zhang Z.-B., Choi C.-S., 2008, A\&A, 484, 293

Zhu D., Zinde-Walsh V., 2009, J. Econometrics, 148, 86

Zitouni H., Guessoum N., Azzam W. J., Mochkovitch R., 2015, Ap\&SS, 357,1

This paper has been typeset from a $\mathrm{T}_{\mathrm{E}} \mathrm{X} / \mathrm{L} \mathrm{T} \mathrm{X}$ file prepared by the author. 